University of Wollongong

Research Online

Australian Institute for Innovative Materials -

Papers

Australian Institute for Innovative Materials

$1-1-2014$

\title{
Enhanced sodium-ion battery performance by structural phase transition from two-dimensional hexagonal-SnS2 to orthorhombic-SnS
}

Tengfei Zhou

University of Wollongong, tz765@uowmail.edu.au

Wei Kong Pang

University of Wollongong, wkpang@uow.edu.au

Chaofeng Zhang

University of Wollongong, czhang@uow.edu.au

Jianping Yang

University of Wollongong

Zhixin Chen

University of Wollongong, zchen@uow.edu.au

See next page for additional authors

Follow this and additional works at: https://ro.uow.edu.au/aiimpapers

Part of the Engineering Commons, and the Physical Sciences and Mathematics Commons

Research Online is the open access institutional repository for the University of Wollongong. For further information contact the UOW Library: research-pubs@uow.edu.au 


\title{
Enhanced sodium-ion battery performance by structural phase transition from two-dimensional hexagonal-SnS2 to orthorhombic-SnS
}

\begin{abstract}
Structural phase transitions can be used to alter the properties of a material without adding any additional elements and are therefore of significant technological value. It was found that the hexagonal-SnS2 phase can be transformed into the orthorhombic-SnS phase after an annealing step in an argon atmosphere, and the thus transformed SnS shows enhanced sodium-ion storage performance over that of the SnS2, which is attributed to its structural advantages. Here, we provide the first report on a SnS@graphene architecture for application as a sodium-ion battery anode, which is built from twodimensional SnS and graphene nanosheets as complementary building blocks. The as-prepared SnS@graphene hybrid nanostructured composite delivers an excellent specific capacity of $940 \mathrm{mAh}$ $\mathrm{g}-1$ and impressive rate capability of 492 and $308 \mathrm{mAh}$ g- 1 after 250 cycles at the current densities of 810 and $7290 \mathrm{~mA} \mathrm{~g}-1$, respectively. The performance was found to be much better than those of most reported anode materials for $\mathrm{Na}$-ion batteries. On the basis of combined ex situ Fourier transform infrared spectroscopy, X-ray photoelectron spectroscopy, and ex situ X-ray diffraction, the formation mechanism of SnS@graphene and the synergistic Na-storage reactions of SnS in the anode are discussed in detail. The SnS experienced a two-structural-phase transformation mechanism (orthorhombic-SnS to cubic-Sn to orthorhombic-Na3.75Sn), while the SnS2 experienced a three-structural-phase transformation mechanism (hexagonal-SnS2 to tetragonal-Sn to orthorhombic-Na3.75Sn) during the sodiation process. The lesser structural changes of SnS during the conversion are expected to lead to good structural stability and excellent cycling stability in its sodium-ion battery performance. These results demonstrate that the SnS@graphene architecture offers unique characteristics suitable for high-performance energy storage application.
\end{abstract}

\section{Keywords}

hexagonal, sns2, orthorhombic, sns, transition, phase, structural, dimensional, performance, two, battery, ion, sodium, enhanced

\section{Disciplines}

Engineering | Physical Sciences and Mathematics

\section{Publication Details}

Zhou, T., Pang, W. Kong., Zhang, C., Yang, J., Chen, Z., Liu, H. Kun. \& Guo, Z. (2014). Enhanced sodium-ion battery performance by structural phase transition from two-dimensional hexagonal-SnS2 to orthorhombic-SnS. ACS Nano, 8 (8), 8323-8333.

\section{Authors}

Tengfei Zhou, Wei Kong Pang, Chaofeng Zhang, Jianping Yang, Zhixin Chen, Hua-Kun Liu, and Zaiping Guo 


\section{Enhanced Sodium-ion Battery Performance by}

\section{Structural Phase Transition from Two-dimensional}

\section{Hexagonal $\mathrm{SnS}_{2}$ to Orthorhombic $\mathrm{SnS}$}

Tengfei Zhou ${ }^{a, b}$, Wei Kong Pang ${ }^{a, c}$, Chaofeng Zhang ${ }^{a}$, Jianping Yang ${ }^{a}$, Zhixin Chen $^{a}$, Hua Kun Liu ${ }^{a}$, and Zaiping Guo ${ }^{a, b^{*}}$

a Institute for Superconducting and Electronic Materials, School of Mechanical, Materials and Mechatronics Engineering, University of Wollongong, North Wollongong, NSW 2500, Australia. Email: zguo@uow.edu.au

b Hubei Collaborative Innovation Center for Advanced Organic Chemical Materials, College of Chemistry and Chemical Engineering, Hubei University, Wuhan 430062, P.R. China.

c Australian Nuclear Science and Technology Organization, Locked Bag 2001, Kirrawee DC, New South Wales 2232, Australia

Keywords: Sodium-ion battery, NIB, SIB, SnS, $\mathrm{SnS}_{2}$, Sn, Graphene, Nanosheets, Anode 


\section{Abstract}

Structural phase transitions can be used to alter the properties of a material without adding any additional elements and are therefore of significant technological value. It was found that the hexagonal$\mathrm{SnS}_{2}$ phase can be transformed into orthorhombic-SnS phase after an annealing step in an argon atmosphere, and the thus transformed SnS shows enhanced sodium ion storage performance over that of the $\mathrm{SnS}_{2}$, which is attributed to its structural advantages. Here, we provide the first report on an SnS@graphene architecture for application as sodium ion battery anode, which is built from twodimensional (2D) SnS and graphene nanosheets as complementary building blocks. The as-prepared SnS@graphene hybrid nanostructured composite delivers excellent specific capacity of $940 \mathrm{mAh} \mathrm{g}^{-1}$ and impressive rate capability of 492 and $308 \mathrm{mAh} \mathrm{g}^{-1}$ after 250 cycles at the current densities of 810 and $7290 \mathrm{~mA} \mathrm{~g}^{-1}$, respectively. The performance was found to be much better than those of most reported anode materials for Na ion batteries. Based on combined ex-situ Fourier transform infrared spectroscopy, X-ray photoelectron spectroscopy, and ex-situ X-ray diffraction, the formation mechanism of SnS@graphene and the synergistic Na-storage reactions of SnS in the anode are discussed in detail. The SnS experienced a two-structural-phase transformation mechanism (orthorhombic-SnS to cubic-Sn to orthorhombic- $\mathrm{Na}_{3.75} \mathrm{Sn}$ ), while the $\mathrm{SnS}_{2}$ experienced a three-structural-phase transformation mechanism (hexagonal-SnS 2 to tetragonal-Sn to orthorhombic- $\mathrm{Na}_{3.75} \mathrm{Sn}$ ) during the sodiation process. The lesser structural changes of SnS during the conversion are expected to lead to good structural stability and excellent cycling stability in its sodium ion battery performance. These results demonstrate that the SnS@graphene architecture offers unique characteristics suitable for high-performance energy storage application. 


\section{Introduction}

Bestowed by its similar chemical nature to $\mathrm{Li}$, the Na-ion battery has recently again attracted increasing attention for large-scale energy storage in renewable energy and smart grid applications, because of both the low cost associated with its high natural abundance and the decent energy densities. ${ }^{1-5}$ Many electrode materials for the Li-ion chemistry have been investigated as drop-in replacements for Na-ion batteries. ${ }^{6}$ Most such efforts have been ineffective, however, as evidenced by low capacity utilization, inferior rate capability, poor cycling stability, or even complete electrochemical inactivity, for which the larger size of the Na-ion relative to the Li-ion is generally believed to be responsible. ${ }^{7}$

Among the limited number of Na-ion battery anode materials, tin (Sn) is a promising high theoretical capacity material with theoretical capacity of $847 \mathrm{~mA} \mathrm{~h} \mathrm{~g}{ }^{-1}\left(\mathrm{Na}_{15} \mathrm{Sn}_{4}\right){ }^{8}$. The large volume expansion $(\sim$ $420 \%$ ) of Sn during sodiation, however, essentially prevents access to this capacity. ${ }^{9,} 10$ To circumvent these issues, studies on the preparation of composites containing carbon based materials and other metals as well as tin were carried out. For example, Xiao et al. developed nanosized SnSb/C to dilute the opportunities for Sn aggregation, which achieved an unprecedented capacity of $435 \mathrm{~mA} \mathrm{~h} \mathrm{~g}{ }^{-1}{ }^{11} \mathrm{Xu}$ et al. have produced porous carbon in a composite with tin, and they have tested it as anode for both sodium and lithium ion batteries. ${ }^{9}$ Recently, metal sulfides have been intensively studied as alternative candidates for commercial anode materials because of their higher capacities. ${ }^{12-18}$ And it was found that the electrochemical properties of layered sulfides $\left(\mathrm{SnS}_{2}, \mathrm{MoS}_{2}, \mathrm{WS}_{2}\right)$ can be further improved by integration with graphene ${ }^{18-23}$. Among the various metal sulfide anodes, orthorhombic-SnS has a unique layered structure with a large interlayer spacing ( $c=0.4330 \mathrm{~nm}$, space group Pnma), so that this crystallographic feature makes it suitable for the intercalation of $\mathrm{Na}$ ions and compensation of the alloying/de-alloying volume changes. ${ }^{24,25}$ In addition, the arrangement of Sn atoms in SnS were much 
similar to that of cubic-Sn, which is the phase after sodiation, expecting a good structural stability and excellent cycling stability for the performance on the sodium ion battery. Moreover, the layered structure of SnS should also be viable for reversible $\mathrm{Na}^{+}$storage. Exfoliation of these materials into layer sheets largely preserves their properties and also leads to additional characteristics due to confinement effects. ${ }^{26-30}$ The intralayer Sn-S bonds are predominantly covalent in nature, whereas the layers are coupled by weak van der Waals forces, thus allowing the crystal to readily cleave along the layer surface. ${ }^{31}$ Studies have shown that the analogous single layers are stabilized by the development of a ripple structure, as in the case of graphene. ${ }^{32,33}$ Furthermore, graphene sheets can easily self-assemble into conductive networks, not only offering a large number of accessible open pores to $\mathrm{Na}^{+}$in the electrolyte, but also allowing for the growth and anchoring of functional inorganic nanomaterials at high loading. ${ }^{9,} 34,35$ In addition to the composition and arrangement of atoms in materials, dimensionality also plays a crucial role in determining their fundamental properties. ${ }^{36}$ This has been most strikingly highlighted over the past few years with two-dimensional (2D) graphene. ${ }^{15-17,}$, 37-39 For electrode materials, to completely utilize the 2D nature of graphene, an ideal second phase should be 2D as well, because the diffusion time $\left(t=L^{2} / D\right)$ of ions in electrode materials is proportional to the square of the diffusion length, $L$ and inversely proportional to the diffusion coefficient, $D$, so that a filmy 2D structure with shorter diffusion length will significantly improve the diffusion efficiency of alkali metal ions and thus enhance the rate performance of sodium/lithium ion batteries. ${ }^{1,35,37,40}$

Herein, we demonstrate a simple hydrothermal and annealing approach to construct hybrid architectures by employing 2D nanosheets of $\mathrm{SnS}$ and graphene as complementary building blocks via their controllable assembly properties. The resulting SnS@graphene architecture offers unique characteristics needed for high-performance electrodes. For electrical conductivity, such a structure enables locally intimate contact between the SnS and the graphene, which ensures excellent network conductivity 
throughout the whole electrode. For ion transport, the porous structure provides numerous channels that allow access to the electrolyte, while the active framework made from filmy 2D SnS nanosheets provides a shortened ion-diffusion length. For electrode stability, during the charge/discharge process, the $\mathrm{SnS}$ experienced a two-structural-phase transformation while the $\mathrm{SnS}_{2}$ experienced a threestructural-phase transformation mechanism. That could be largely improving the structural stability and excellent cycling stability of SnS for their performance in the sodium ion battery. And the graphene component, the SnS layer structure, and the porous structure help to buffer the volume changes and stress that are generated during charge and discharge, and thus maintain the structural stability of the whole electrode. As a consequence, the optimal SnS@graphene hybrid architecture exhibits a very high reversible capacity of $940 \mathrm{mAh} \mathrm{g}^{-1}$ and excellent high-rate capability for $\mathrm{Na}$ ion storage. After 250 cycles, the specific capacities are still 492 and $308 \mathrm{mAh} \mathrm{g}^{-1}$, as the current densities are increased to 810 and $7290 \mathrm{~mA} \mathrm{~g}^{-1}$, respectively.

\section{Results and Discussion}

The overall synthetic procedure for the hybrid SnS architecture involves two steps: (1) the preparation of the $\mathrm{SnS}_{2} @ g r a p h e n e$ precursor under hydrothermal conditions (as shown in Scheme 1); and (2) the subsequent structural phase transition process in argon in order to obtain the SnS@graphene hybrid architecture (as shown in Scheme 2). 

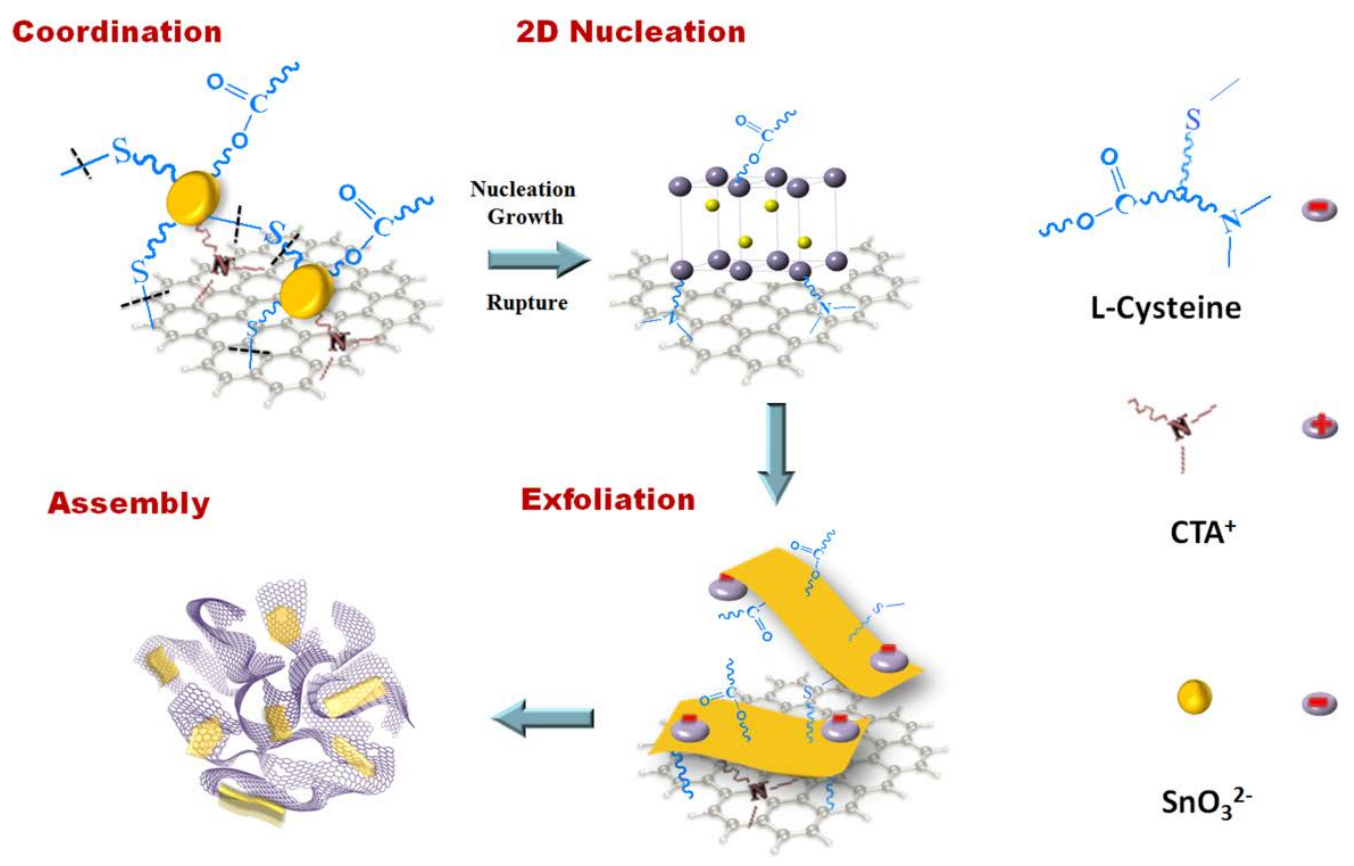

CTA $^{+}$

$\mathrm{SnO}_{3}{ }^{2-}$

Scheme 1. Schematic diagram illustrating the hydrothermal preparation of the hybrid tin sulfide and graphene architecture. Coordination: $\mathrm{CTA}^{+}$adsorbed on the GO due to the electrostatic interaction between the cationic surfactant and the negatively charged $\mathrm{GO}$, then, $\mathrm{SnO}_{3}{ }^{2-}$ could be attached to the $\mathrm{CTA}^{+}-\mathrm{GO}$ surface to form a $\mathrm{SnO}_{3}{ }^{2}-\mathrm{CTA}^{+}-\mathrm{GO}$ complex. 2D Nucleation: In the presence of GO and Lcysteine, the crystal nucleated with the typical 2D nucleation-layer growth (atom color code: purple, Tin; yellow, Sulphur). Exfoliation: The excess L-cysteine can be negatively charged under alkaline solution and make the L-cysteine-capped $\mathrm{SnS}_{2}$ particles repel each other and promote the exfoliation. Assembly: Hybrid architecture formed by the self-assembly of flexible graphene and layered $\mathrm{SnS}_{2}$ nanosheets.

To understand the synthetic procedures, ex-situ Fourier transform infrared (FTIR) spectroscopy was conducted. Two peaks are found at 2914 and $2838 \mathrm{~cm}^{-1}$ in the FTIR spectrum of the SnS $2 @$ graphene nanocomposite [Figure 1(a)], which can be assigned to the $\mathrm{C}-\mathrm{H}$ stretching vibrations of $-\mathrm{CH}_{3}$ and $-\mathrm{CH}_{2}$ in $\mathrm{CTA}^{+}$, respectively, indicating that the $\mathrm{CTA}^{+}$has adsorbed on the GO surface. The adsorption of 
$\mathrm{CTA}^{+}$on GO is due to the electrostatic interaction between the cationic surfactant and the negatively charged $\mathrm{GO},{ }^{35}$ which imparts a positive charge to the GO to overcome the innate charge incompatibility between $\mathrm{GO}$ and $\mathrm{SnO}_{3}{ }^{2-}$. Consequently, $\mathrm{SnO}_{3}{ }^{2-}$ could be attached to the $\mathrm{CTA}^{+}-\mathrm{GO}$ surface to form a $\mathrm{SnO}_{3}{ }^{2-}$-CTA ${ }^{+}$-GO complex. After that, L-cysteine is added into the suspension. L-cysteine is of particular interest to researchers due to its multifunctional groups (-SH, $-\mathrm{NH}_{2}$, and $\left.-\mathrm{COO}^{-}\right)$, which can be used for the conjugation of metallic ions or other functional groups. ${ }^{17,}{ }^{41}$ The intensity of the characteristic N-H deformation and $\mathrm{C}=\mathrm{O}$ stretching vibration of the amide bond observed at $1535 \mathrm{~cm}^{-1}$ and $1657 \mathrm{~cm}^{-1}$, respectively, for pure L-cysteine is weakened in the products, while the doublet peaks at 3350 and $3250 \mathrm{~cm}^{-1}$ from the symmetric vibration of peptide groups in L-cysteine merge into a broad band in the range of $3420-3200 \mathrm{~cm}^{-1}$, suggesting that the nitrogen atom of the amide bond is possibly coordinated to the $\mathrm{SnO}_{3}{ }^{2-}$ ion. ${ }^{16}$

$$
\begin{aligned}
& \mathrm{HSCH}_{2} \mathrm{CHNH}_{2} \mathrm{COOH}+\mathrm{H}_{2} \mathrm{O} \rightarrow \mathrm{CH}_{3} \mathrm{COOH}+\mathrm{NH}_{3}+\mathrm{H}_{2} \mathrm{~S} \\
& \mathrm{SnO}_{3}^{2-}+2 \mathrm{H}_{2} \mathrm{~S}+2 \mathrm{CH}_{3} \mathrm{COOH} \rightarrow \mathrm{SnS}_{2}+3 \mathrm{H}_{2} \mathrm{O}+2 \mathrm{CH}_{3} \mathrm{COO}^{-}
\end{aligned}
$$

In our synthetic procedures, L-cysteine not only plays the role of a reducing agent and sulfur donor, but also acts as a stabilizing agent during the whole hydrothermal process, in which the L-cysteine releases $\mathrm{H}_{2} \mathrm{~S}$ as a sulfide source as well as a reducing agent [Equation (1)], resulting in the $\mathrm{SnO}_{3}{ }^{2-}$ precursor to $\mathrm{SnS}_{2}$ and the reduction of GO to graphene [Equation (2)]. The excess L-cysteine that remains in the solution as a stabilizing agent is a key factor that promotes the exfoliation of the hybrid precursor and enhances the stability of the colloidal dispersion. From the FTIR spectrum of $\mathrm{SnS}_{2} @$ graphene precursor after $1 \mathrm{~h}$ hydrothermal reaction [Figure 1(a)], the peaks at 3124 and $3026 \mathrm{~cm}^{-1}$ for L-cysteine assigned to the $\mathrm{N}-\mathrm{H}$ stretching vibration of the zwitterion (-OOC-C- $\mathrm{NH}_{3}{ }^{+}$) and the peak at $1713 \mathrm{~cm}^{-1}$ assigned to the stretching vibration of the - $\mathrm{COOH}$ group of glycine residue, respectively, also disappear in the products, 
indicating that both the amino and the carboxyl groups of L-cysteine have been deprotonated, and the alkaline solution is generally believed to be responsible for this. ${ }^{42}$ This is because the L-cysteine can be negatively charged under alkaline solution and make the L-cysteine-capped $\mathrm{SnS}_{2}$ particles repel each other. $^{43}$
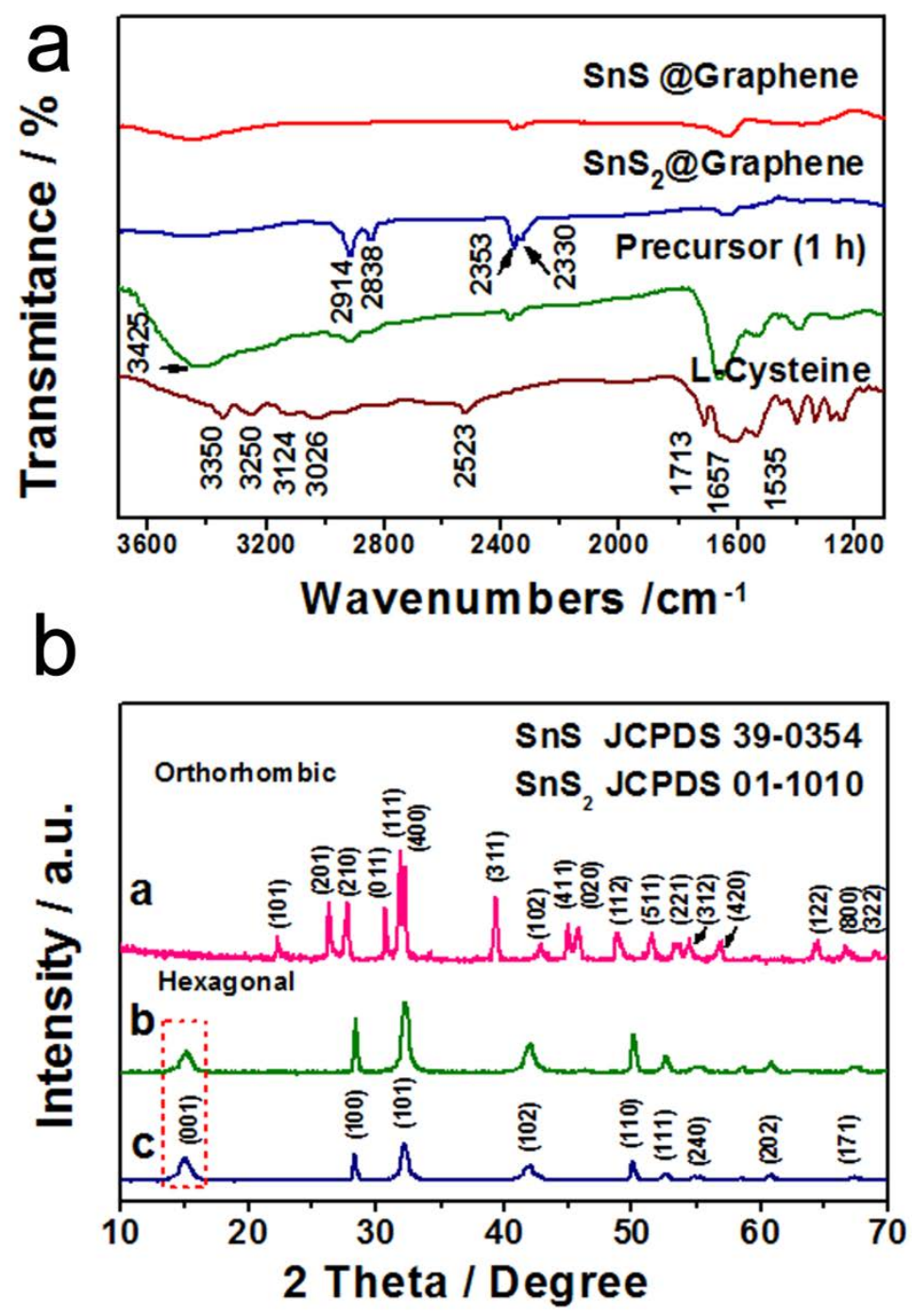

Figure 1. (a) FTIR spectra of SnS@graphene, SnS $2 @$ graphene, SnS $S_{2} @$ graphene precursor after 1h hydrothermal reaction, and L-cysteine. (b) XRD patterns of a) SnS@graphene [orthorhombic SnS (JCPDS No. 39-0354)] and b) $\mathrm{SnS}_{2} @ g r a p h e n e$ architectures, and c) pristine $\mathrm{SnS}_{2}$. The XRD pattern of the $\mathrm{SnS}_{2} @ g r a p h e n e$ composite is similar to that of pristine $\mathrm{SnS}_{2}$ [hexagonal $\mathrm{SnS}_{2}$ (JCPDS No. 01-1010)], 
the intensity of (001) plane is much lower than in the case of pristine $\mathrm{SnS}_{2}$, indicating that growth of the $\mathrm{SnS}_{2}$ was inhibited in the hybrid due to the intervention of graphene and that a layered/sheet-like structure of $\mathrm{SnS}_{2}$ was formed in the hybrid architecture.

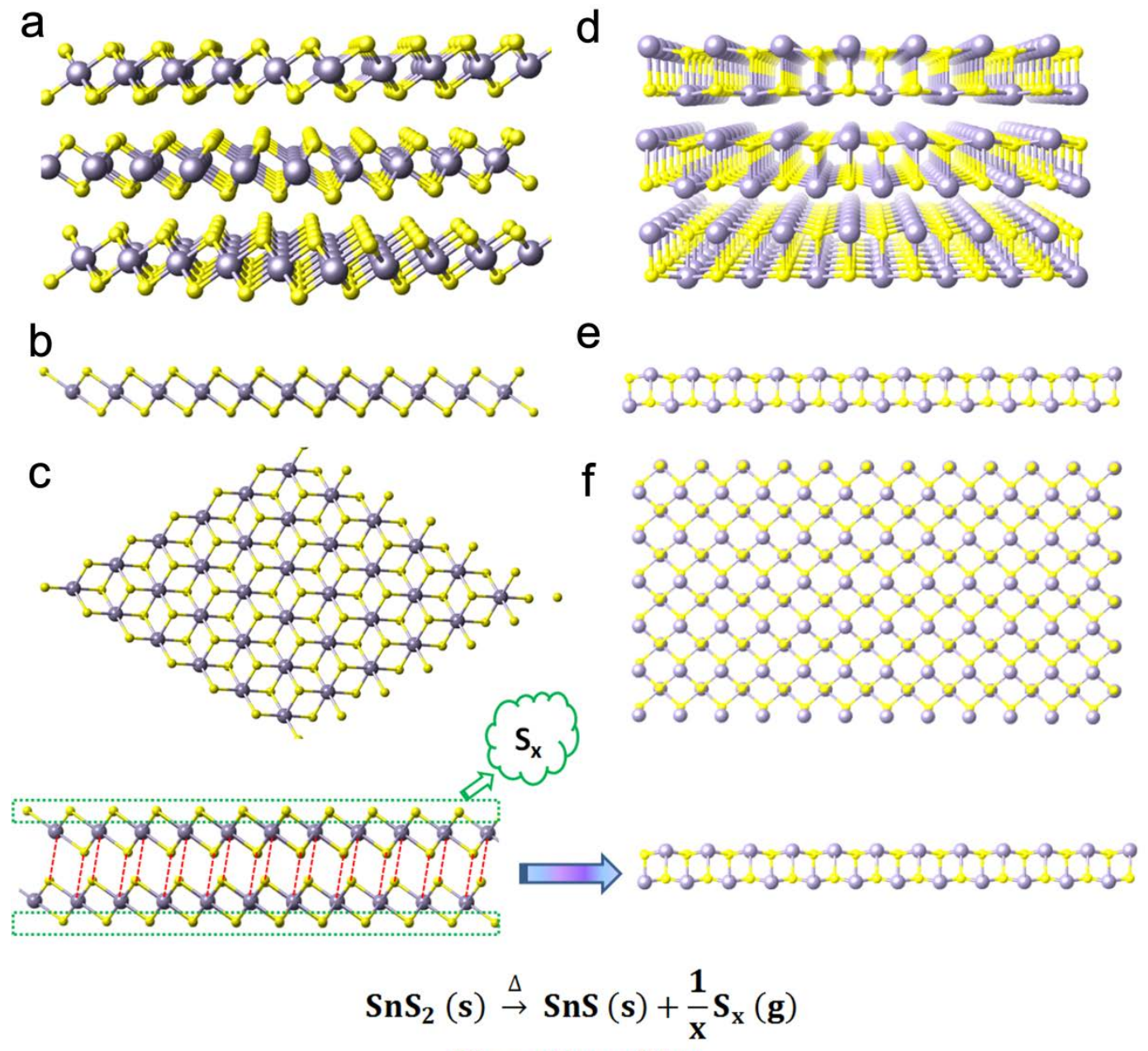

Phase Transition

SnS $_{2}$ Hexagonal $\longrightarrow$ Orthorhombic SnS

Scheme 2. Schematic diagram (bottom) illustrating the phase transition process between $\mathrm{SnS}_{2}$ and $\mathrm{SnS}$.

The crystal structures of hexagonal $\mathrm{SnS}_{2}(\mathrm{a}, \mathrm{b}, \mathrm{c})$ and orthorhombic $\mathrm{SnS}$ (d, e, f) as shown in different sectional views (atom color code: purple, Tin; yellow, Sulphur). The formation of orthorhombic SnS phase may rely on the $S_{x}$ depletion in a later stage of the phase transformation process. After annealing, the $\mathrm{SnS}_{2}$ dissociation occurs, $\mathrm{S}$ depletion and high temperatures promote dissociation processes. This 
route is based on the incongruent sublimation of the $\mathrm{SnS}_{2}$ and proposes the direct formation of the $\mathrm{SnS}$ from $\mathrm{SnS}_{2}$ through the proposed reaction in the scheme.

The subsequent structural phase transition process in argon would finalize the synthesis of the SnS@graphene architecture. The possible dissociation reaction at high temperature is proposed in the equation shown in Scheme 2. ${ }^{15,44}$ Figure 1(b) shows a representative X-ray diffraction (XRD) pattern of pristine $\mathrm{SnS}_{2}$. All of the diffraction peaks can be indexed to hexagonal $\mathrm{SnS}_{2}$ (JCPDS No. 01-1010) [Scheme 2 a,b and c], indicating that pure, single-phase, and well crystallized $\mathrm{SnS}_{2}$ has been obtained. The XRD pattern of the $\mathrm{SnS}_{2} @$ graphene composite is similar to that of pristine $\mathrm{SnS}_{2}$, except for the (001) plane of the $\mathrm{SnS}_{2}$ [Figure 1b (b, c)], the intensity of which is much lower than in the case of pristine $\mathrm{SnS}_{2}$, indicating that growth of the $\mathrm{SnS}_{2}$ (001) plane was inhibited in the hybrid due to the intervention of graphene and that a layered/sheet-like structure of $\mathrm{SnS}_{2}$ was formed in the hybrid architecture. ${ }^{14}$ As shown in Figure 1(b), after annealing, an entirely new and well crystallized phase of orthorhombic SnS (space group Pbnm (62), JCPDS No. 39-0354) can be observed from the XRD pattern [Scheme $2 \mathbf{d}, \mathbf{e}$ and f]. The formation of this phase may rely on the $S$ depletion in a later stage of the phase transformation process. Two intermediate processes could be involved in the synthesis of SnS [Scheme 2]. Initially, in the high $S$ availability regime, the $\mathrm{SnS}_{2}$ dissociation occurs. After that, $\mathrm{S}$ depletion and high temperatures promote dissociation processes. The chalcogen depletion occurs mainly by localized film segregation and by condensation in other parts of the furnace and exhaust system. ${ }^{45}$ This route is based on the incongruent sublimation of the $\mathrm{SnS}_{2}$ and proposes the direct formation of the $\mathrm{SnS}$ from $\mathrm{SnS}_{2}$ through the following reaction: ${ }^{46}$

$$
\operatorname{SnS}_{2}(s) \stackrel{\Delta}{\rightarrow} \operatorname{SnS}(s)+\frac{1}{x} S_{x}(g)
$$


where $x=2-8$, which represent the various $\mathrm{S}$ species that can be formed during this process. In order to measure the content of graphene in the as-synthesized SnS@graphene composite, thermogravimetric analysis (TGA) was carried out from 50 to $800^{\circ} \mathrm{C}$ in air (Figure S2). From the TGA curve, the major weight loss from 450 to $600{ }^{\circ} \mathrm{C}$ was due to the removal of graphene by the oxidation process, and it can be calculated that the SnS@graphene composite contains 13.8\% graphene.

XPS analysis was also conducted to investigate the surface electronic states and the chemical composition of the as-synthesized sample (Figure S1 in the Supporting Information). Compared with the spectra of SnS ${ }_{2} @ G r a p h e n e$, the Sn 3d peaks of SnS@Graphene are shifted toward lower binding energy by $0.7 \mathrm{eV}\left(3 \mathrm{~d}_{5 / 2}\right)$ without any obvious change in the spectral shape. This phenomenon results from the large difference in electronegativity between coordinating groups, which confirms that the evolution of the process goes through a transformation from the $\mathrm{SnS}_{2}$-ligand complexes to SnS. From Figure S1(c), it can be seen that the asymmetrical S 2p spectrum for the $\mathrm{SnS}_{2} @$ Graphene, can be divided into three peaks, indicating that there are three chemical environments. The peaks at $162.3 \mathrm{eV}$ and $163.7 \mathrm{eV}$ could be attributed to the binding energies of $S 2 p_{3 / 2}$ and $S 2 p_{1 / 2}{ }^{34}$. The other peak at $165.1 \mathrm{eV}$ could be assigned to the binding energy of sulfur in the thiol group, which is in agreement with the above FTIR analysis $^{41}$. 


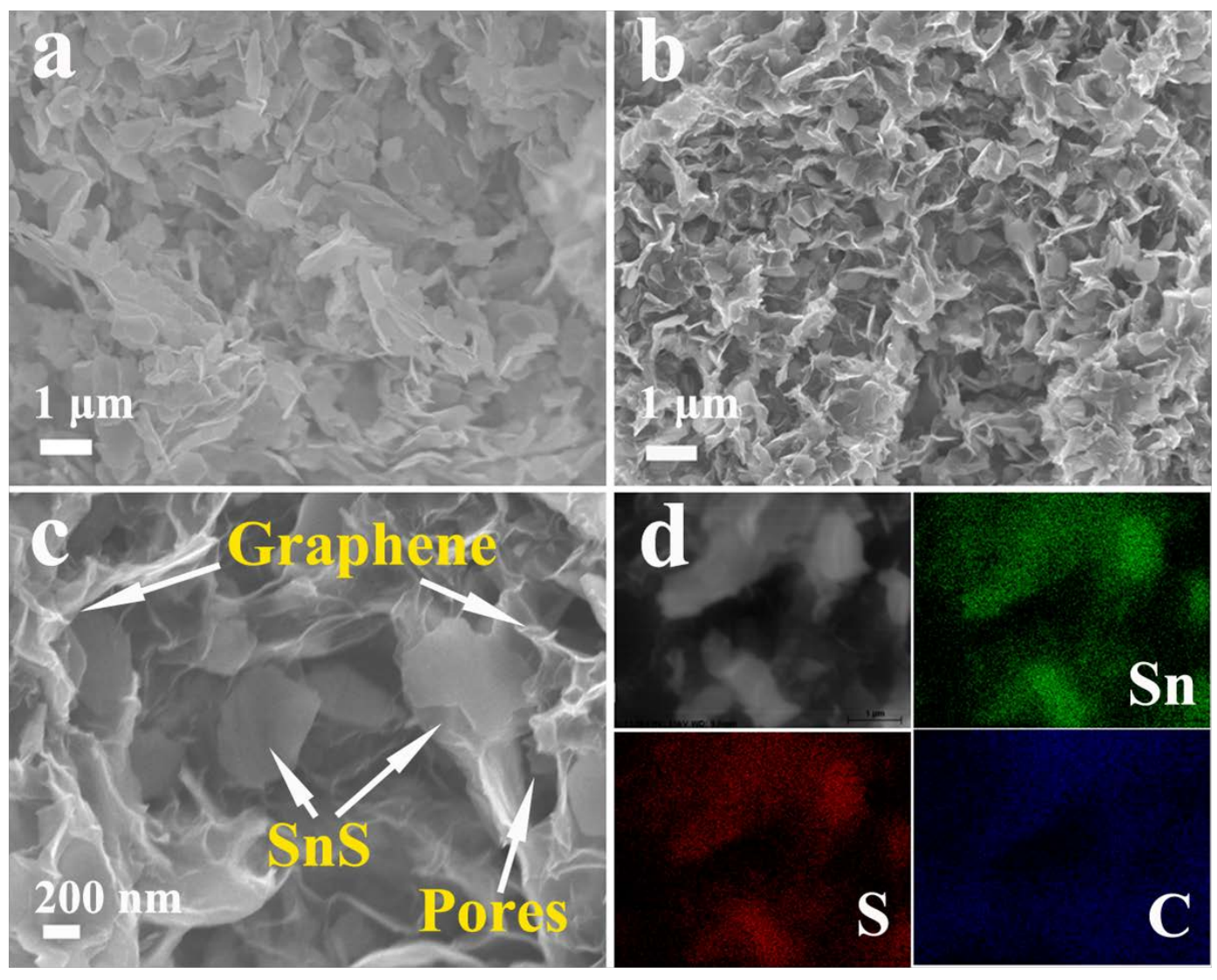

Figure 2. SEM image of hybird $\mathrm{SnS}_{2} @$ graphene (a); (b, c) typical SEM images of SnS@graphene architecture at different magnifications, which reveal the porous structure and thin walls containing both SnS and graphene nanosheets. (d) Element mapping images of the SnS@graphene with corresponding SEM image. 


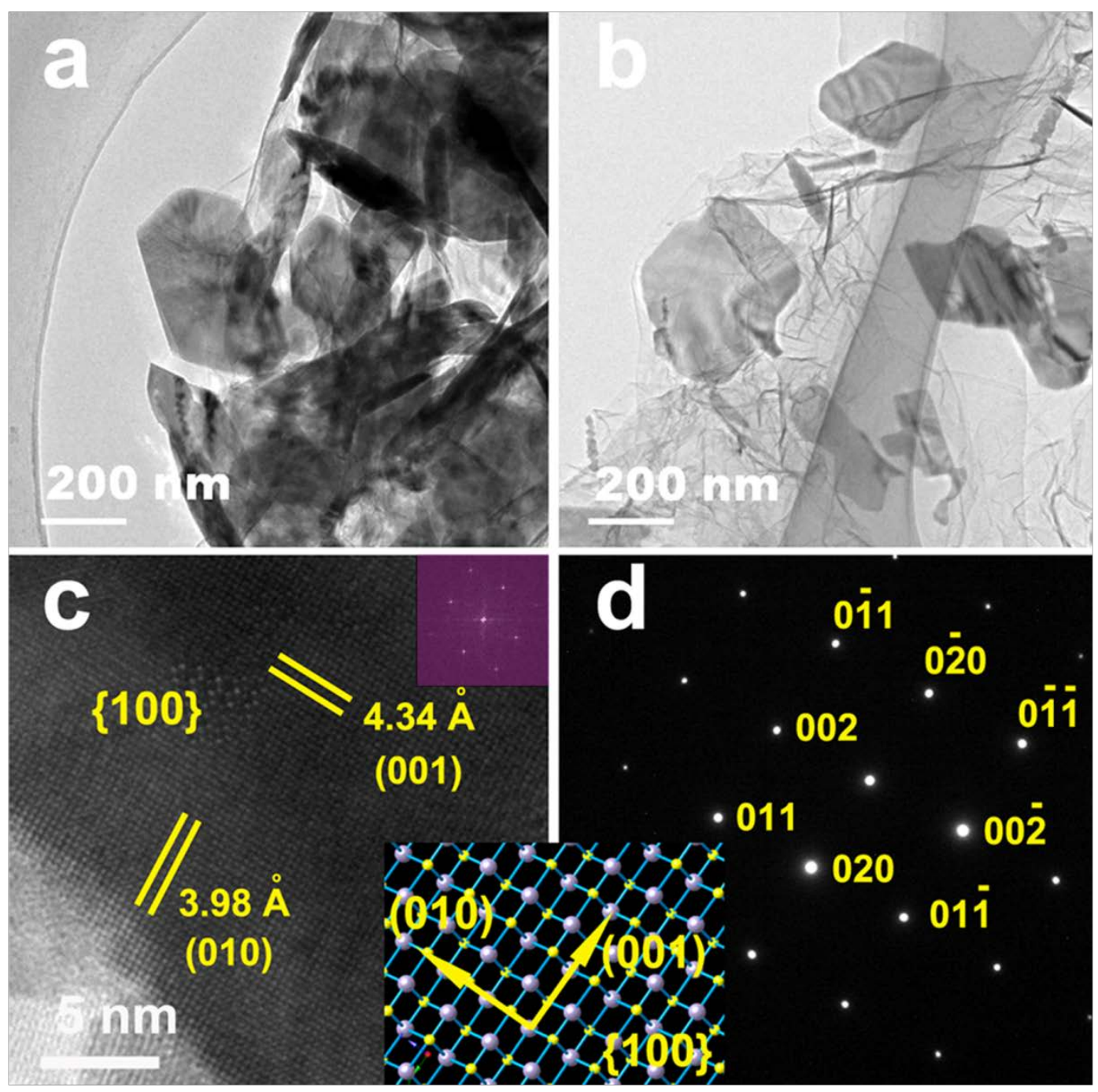

Figure 3. Representative TEM images of (a) $\mathrm{SnS}_{2} @$ graphene and (b) SnS@graphene. (c) HRTEM image of SnS with the resulting FFT pattern (inset), and (d) the corresponding SAED pattern, which can be indexed to SnS (100). The inset between (c) and (d) shows the atomistic model of the SnS (100) surface. 
The morphology and microstructure of the as-prepared SnS ${ }_{2} @$ graphene and SnS@graphene composites were investigated via field emission scanning electron microscopy (SEM) and transmission electron microscopy (TEM). The GO sheets serve as the substrate for the nucleation and growth of $\mathrm{SnS}_{2}$ into a layered structure, due to the interaction between the GO with functional groups and the $\mathrm{SnO}_{3}{ }^{2-}$ precursor. On the other hand, $\mathrm{GO}$ in the composite also could restrain the stacking of $\mathrm{SnS}_{2}$ in the $c$-direction to promote the formation of $\mathrm{SnS}_{2}$ sheet-like structures. ${ }^{14,47,48}$ With no GO in the synthetic process, the pristine $\mathrm{SnS}_{2}$ materials mainly consist of irregular nanoparticles with a small quantity of sheet-like structures (Figure S3). Abundant $\mathrm{SnS}_{2}$ nanosheets are clearly obtained after GO is included in the synthetic process [Figure 2(a) and Figure 3(a)]. From SEM observations, we found that the SnS nanosheets [Figure 2(c)] are more rigid than the graphene nanosheets. ${ }^{38}$ As shown in the SEM [Figure 2(b, c)] and TEM [Figure 3(b)] images, the as-prepared SnS@graphene architecture possesses a hybrid structure with interconnected pores, ranging from several nanometers to several micrometers in size. Moreover, the thin and rigid SnS nanosheets can be clearly observed and are homogeneously confined in the framework, which efficiently hampers their aggregation and restacking, as we expected. The partial overlapping or coalescing of flexible nanosheets is likely to have originated from the cross-linking of the functional groups in the graphene sheets, and thus, the SnS nanosheets are much easier to efficiently combine with graphene. Importantly, only 2D nanosheets were observed in this sample, indicating a nearly complete 2D morphological yield. Wide area energy dispersive spectroscopy (EDS) analysis indicated a nearly 1:1 atomic ratio of Sn to S (52 \% Sn, 48 \% S) for SnS and 1:2 for SnS 2 (35 \% Sn, 65 \% S), confirming the SnS and $\mathrm{SnS}_{2}$ stoichiometry (Figure S4). Analysis of an individual sheet by selected area electron diffraction (SAED) [Figure 3(d)] indicated that it is single-crystalline and can be indexed to the (100) plane of SnS. ${ }^{31}$ The high resolution TEM (HRTEM) image of the nanosheets [Figure 3(c)] confirms their single-crystalline nature. Measurements of the reciprocal space distances between the fast 
Fourier transform (FFT) diffraction spots [inset image in Figure 3(c)] were quantitatively consistent with those of the SAED pattern. The interatomic distances were determined to be 3.98 and $4.34 \AA$, respectively, corresponding to the (010) and (001) planes, and they match well with the $b$ and $c$ lattice constants of orthorhombic GeS-type SnS [inset image between Figure 3(c) and 3(d)] (b=3.98 $\AA, c=$ $4.33 \AA$ ). Element mapping of the SnS@graphene composite demonstrates that the carbon, tin, and sulphur are homogeneously distributed in the sample and that the 2D SnS nanosheets have been very effectively combined with the graphene nanosheets [Figure 3(d)]. 


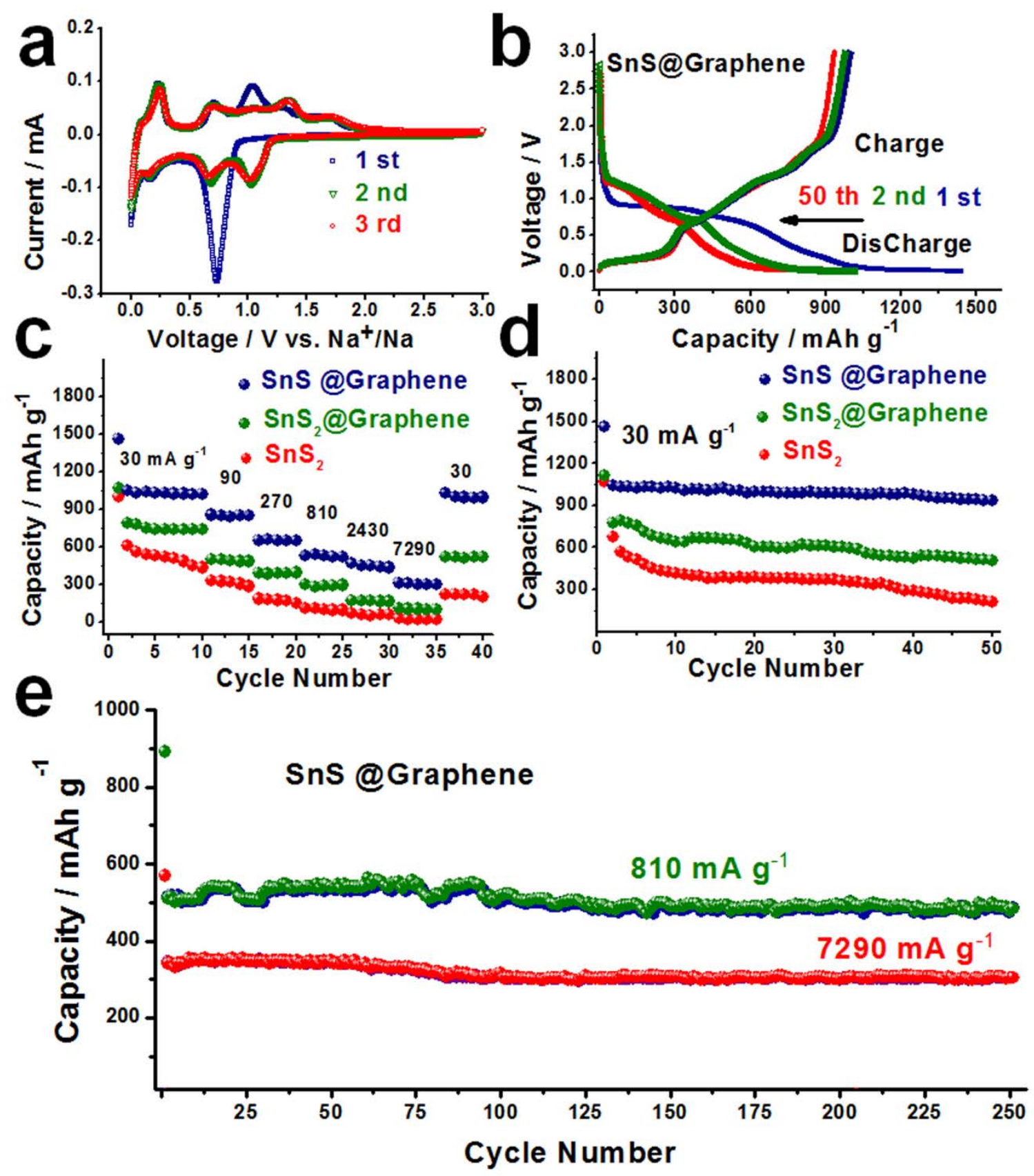

Figure 4. Electrochemical performance of SnS@graphene hybrid architecture for high-performance sodium storage. (a) Cyclic voltammograms for the first 3 cycles of SnS@graphene electrode at a scanning rate of $0.1 \mathrm{mV} \mathrm{s}^{-1}$. (b) Galvanostatic discharge-charge profiles for selected cycles of SnS@graphene electrode at a current density of $30 \mathrm{~mA} \mathrm{~g}^{-1}$. Comparisons of (c) rate capabilities and (d) 
cycling performances of SnS@graphene, SnS ${ }_{2} @$ graphene, and $\mathrm{SnS}_{2}$ electrodes, and (e) long cycling performances of SnS@graphene electrode at different current densities.

The sodium ion storage behaviour of the composites was first characterized by cyclic voltammetry (CV) [Figure 4(a)]. During the first negative scan, a strong reduction peak appeared at $\sim 0.74 \mathrm{~V}$ and disappeared at the second negative scan, corresponding to the decomposition of electrolyte to form the solid-electrolyte interphase (SEI) film. Thus, after the first cycle, three pairs of oxidation/reduction peaks at $0.08 \mathrm{~V} / 0 \mathrm{~V}, 0.24 \mathrm{~V} / 0.15 \mathrm{~V}$, and $0.70 \mathrm{~V} / 0.66 \mathrm{~V}$ clearly emerged and then remained steady, representing the Na alloying/de-alloying reactions. ${ }^{49}$ The peaks at $1.32 \mathrm{~V} / 1.15 \mathrm{~V}$ in the CV curves can be attributed to the conversion reaction with sulphur atoms in the material, and the obvious platform represented by the charge-discharge plateaus also could confirm that the reaction between SnS and Sn is reversible. There is no obvious loss of area in the successive cycles, proving the good cycling stability of the composite. In the CV curves, it is worth noting that all the redox peaks of SnS@graphene composite are well matched to the charge-discharge plateaus [Figure 4(b)]. And the $\mathrm{SnS}_{2} @$ graphene were much different from that of SnS@graphene, there is no plateau around 1.32 V/ $1.15 \mathrm{~V}$ in the curves of $\mathrm{SnS}_{2} @ g$ graphene, suggest that the reaction between $\mathrm{SnS}_{2}$ and $\mathrm{Sn}$ is irreversible [Figure S5 and S6]. The basic reversible reactions of the composite are elucidated by Equations (4) and (5).

$$
\begin{aligned}
& \mathrm{SnS}+2 \mathrm{Na}^{+}+2 \mathrm{e}^{-} \leftrightarrow \mathrm{Na}_{2} \mathrm{~S}+\mathrm{Sn} \\
& 4 \mathrm{Sn}+15 \mathrm{Na}^{+}+15 \mathrm{e}^{-} \leftrightarrow \mathrm{Na}_{15} \mathrm{Sn}_{4}
\end{aligned}
$$

The electrochemical performance of the hybird SnS@graphene architecture was systematically evaluated by galvanostatic discharge-charge measurements. Remarkably, a very high capacity of 1437 $\mathrm{mAh} \mathrm{g}^{-1}$ is achieved in the initial cycle at a current density of $30 \mathrm{~mA} \mathrm{~g}^{-1}$, in the case of the SnS@graphene architecture [Figure 4(c, d)]. Even after 50 cycles, both the discharge and the charge 
capacities of this architecture are stable at about $940 \mathrm{mAh} \mathrm{g}^{-1}$, delivering $92.4 \%$ of the $2^{\text {nd }}$ cycle capacity (1037 $\mathrm{mAh} \mathrm{g}^{-1}$ ) [Figure 4(d)]. It should be noted that a large capacity of nearly $1400 \mathrm{~mA} \mathrm{~h} \mathrm{~g}^{-1}$ is obtained for the SnS@Graphene electrode during the first discharge and charge processes. This may be partly attributed to both the presence of defects in the graphene and the disorder of the unique 2D structure of SnS. Moreover, the reversible formation/decomposition of the polymeric gel-like film on the surface of the active materials also contributed to the extra capacity, as reported elsewhere. ${ }^{37,39,48}$ And in addition to the capacity comes from the alloying process of Sn, the reversible reaction between SnS and Sn also contributed to the capacity. (It is an interesting point as well for future studies to understand the reasons why extra sodium ions could be inserted into this hybrid material, and the relevant work is in progress.) These results are in stark contrast to the pure $\mathrm{SnS}_{2}$ and the $\mathrm{SnS}_{2} @$ graphene architecture, which show continuous and progressive capacity decay as cycling progresses. More importantly, the asprepared SnS@graphene architecture exhibits good high-rate performance. On increasing the current density to 810 and $7290 \mathrm{~mA} \mathrm{~g}^{-1}$ [Figure 4(e)], the specific capacities at these current densities are still 492, and $308 \mathrm{mAh} \mathrm{g}^{-1}$ after 250 cycles, respectively. It is instructive to compare the performance of the SnS@graphene electrodes with the best systems reported in scientific literature. Table S1 compares our results with the state-of the art in previously published research on Carbon, $\mathrm{Sn}, \mathrm{SnO}_{2}, \mathrm{SnS}_{2}, \mathrm{Sn}_{4} \mathrm{P}_{3}$ and $\mathrm{MoS}_{2}$-based sodium ion battery anode materials. The performance of our prepared SnS@graphene architecture was found to be much better than those of most reported anode materials for $\mathrm{Na}$ ion batteries. 


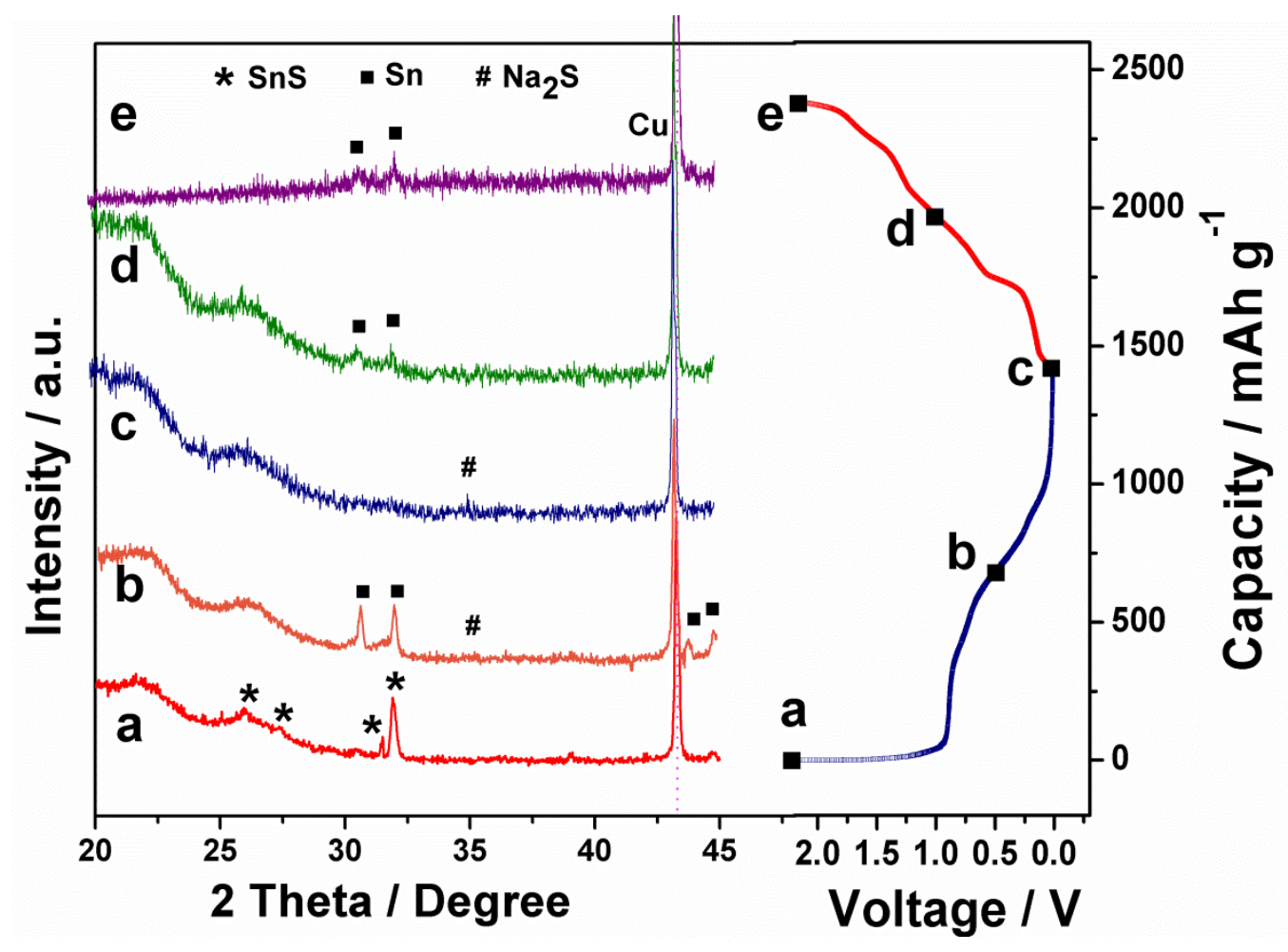

Figure 5. Ex-situ XRD patterns of the SnS@graphene electrode collected at various points as indicated in the corresponding voltage profile: (a) fresh electrode, (b) after $1^{\text {st }}$ discharge to $0.5 \mathrm{~V}$, (c) after $1^{\text {st }}$ discharge to $0.01 \mathrm{~V}$, (d) after $1^{\text {st }}$ charge to $1.0 \mathrm{~V}$, and (e) after $1^{\text {st }}$ charge to $2.2 \mathrm{~V}$.

In order to further clarify the structural changes upon sodiation/desodiation, ex situ XRD analysis of the SnS@graphene was performed, and it revealed that the reversible sodiation/desodiation of SnS proceeded in a two-phase reaction, as shown in Figure 5. The uncharged anode showed a clear XRD pattern of the SnS lattice [Figure 5(a)]. Upon sodiation to $0.5 \mathrm{~V}$, the intensity of the XRD peaks corresponding to SnS decreased [Figure 5(b)], while the main reflections of Sn were observed, implying that the SnS lattice had transformed to metallic $\mathrm{Sn}$ and $\mathrm{Na}_{2} \mathrm{~S}$ phases at an initial stage of the Na insertion reaction, although no obvious $\mathrm{Na}_{2} \mathrm{~S}$ peaks were observed, which indicates that an amorphous $\mathrm{Na}_{2} \mathrm{~S}$ phase was formed during sodiation, while nanocrystalline $\mathrm{Na}_{x} \mathrm{Sn}$ was not detectable by XRD owing to its very small crystallite size. When completely charged to a terminal potential of $0.01 \mathrm{~V}$ [Figure 5(c)], the $\mathrm{Na}_{2} \mathrm{~S}$ 
signal became stronger, and the Sn signal disappeared, indicating that all of the Sn can participate in the Na-alloying reaction by the end of charge process. On desodiation, the intensity of the XRD peaks corresponding to Sn gradually increased [Figure 5(d, e)], indicating that the conversion reaction of Sn to $\mathrm{Na}_{2} \mathrm{~S}$ and $\mathrm{Na}_{x} \mathrm{Sn}$ was reversible.

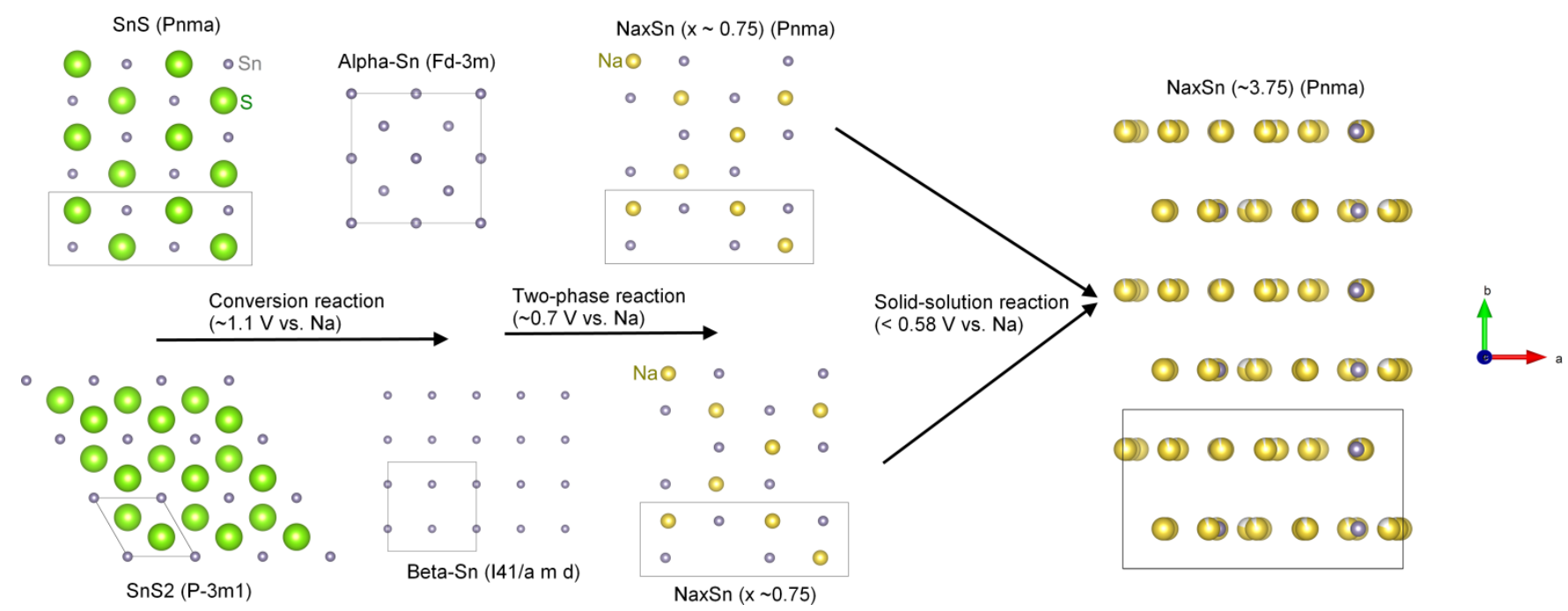

Scheme 3. Schematic illustration of the structure evolution of orthorhombic-SnS and hexagonal-SnS 2 during the sodiation.

By combining the charge-discharge profiles [Figure 4(a, b, d) and Figures S5 and S6] and the ex-situ X-ray diffraction study (Figure 5), we here propose a mechanism to illustrate the discharge process of SnS, which differs from that reported for $\mathrm{SnS}_{2} \cdot{ }^{19}$ During discharging, the SnS anode is first reduced by Na ions through a conversion process, as described in Equation (4), resulting a flat plateau at 1.1 V (vs. $\mathrm{Na}$ ). Alongside the S removal, the orthorhombic structure turns into a face-centered symmetry and forms alpha-Sn. ${ }^{50}$ On further discharging to $0.7 \mathrm{~V}$ (vs. Na), sodium ions intercalate into the Sn structure through a two-phase reaction and occupy the 4c site, which was the original site for S, forming an orthorhombic structure of $\mathrm{Na}_{x} \mathrm{Sn}$ alloy, which is isostructural to the original $\mathrm{SnS}$, and the two-phase reaction results in a short plateau at around $0.7 \mathrm{~V}$ (vs. Na). As discharging continues down to $0.01 \mathrm{~V}$ (vs. 
$\mathrm{Na}$ ), sodium ions occupy the other 4c sites via a solid-solution reaction, forming a sloping plateau-like feature. ${ }^{8}$ The Na intercalation induces internal atomic rearrangement to minimize the strain and gradually form a huge orthorhombic structure, as recorded in ICSD\#105168. On the other hand, hexagonal-SnS 2 transforms into beta-Sn with tetragonal structure and is followed by another two-phase reaction, ${ }^{50}$ changing into orthorhombic $\mathrm{Na}_{3.75} \mathrm{Sn}$, so that it experiences a large lattice volume change (324\% relative to $\mathrm{SnS}_{2}$ ). The major difference between the discharging of $\mathrm{SnS}$ and that of $\mathrm{SnS}_{2}$ is the formation of Sn after the conversion reaction. XRD patterns were obtained for $\mathrm{SnS}$ and $\mathrm{SnS}_{2}$ electrodes after discharge/charge cycles (Figure 6). The stronger peak at $2 \theta=43^{\circ}$ is attributed to the $\mathrm{Cu}$ foil electrode. The $\mathrm{SnS}_{2}$ shows peaks at $30.47^{\circ}$ and $31.90^{\circ}$ corresponding to the (200) and (101) planes of beta-Sn. The alpha-Sn becomes the dominant phase for the SnS, as shown in the XRD pattern of the cell after matching to the (111) and (220) planes of alpha-Sn (JCPDS No. 87-0794; a = $6.489 \AA$ ). It can retain its crystallinity over repeated cycles, contributing to the excellent cycling performance. The total energy ( $E_{\text {tot }}$, total energy of the most stable configuration of reaction products) difference between betaSn andorthorhombic- $\mathrm{Na}_{3.75} \mathrm{Sn}$ is $7.66 \mathrm{eV}$, higher than that of alpha-Sn $(7.125 \mathrm{eV}) .{ }^{50,51}$ This is particularly important, as the alloying of $\mathrm{Na}$ with cubic-Sn (alpha) to form orthorhombic $\mathrm{Na}_{x} \mathrm{Sn}$ is easier than for tetragonal-Sn (beta), allowing higher intercalation capacity [Figure 4(d)] as well as better rate capability [Figure 4(c)]. The whole discharging process involves lattice volume change [242\% relative to SnS, smaller than that of Sn (420\%)]; the structural change during conversion is two-phase, and the solid-solution reactions are smooth, leading us to expect good structural stability and excellent cycling stability, which agrees well with Figure 4(d). Compared to the Sn based anode materials, the potential advantages of SnS over Sn for Na insertion are: higher theoretical capacity than Sn because of the lower mass of the $\mathrm{S}$ atoms, as well as an expected improvement in mechanical stability due to smaller volume change during charge and discharge from the sulphides. ${ }^{52}$ Moreover, sulphide formation is typically 
more reversible than oxide formation, resulting in a higher first cycle efficiency than for oxide materials.

${ }^{51}$ From the perspective of structural stability during cycling, there is no doubt that SnS performs better than $\mathrm{SnS}_{2}$ and $\mathrm{Sn}$ as an anode in the sodium ion battery.

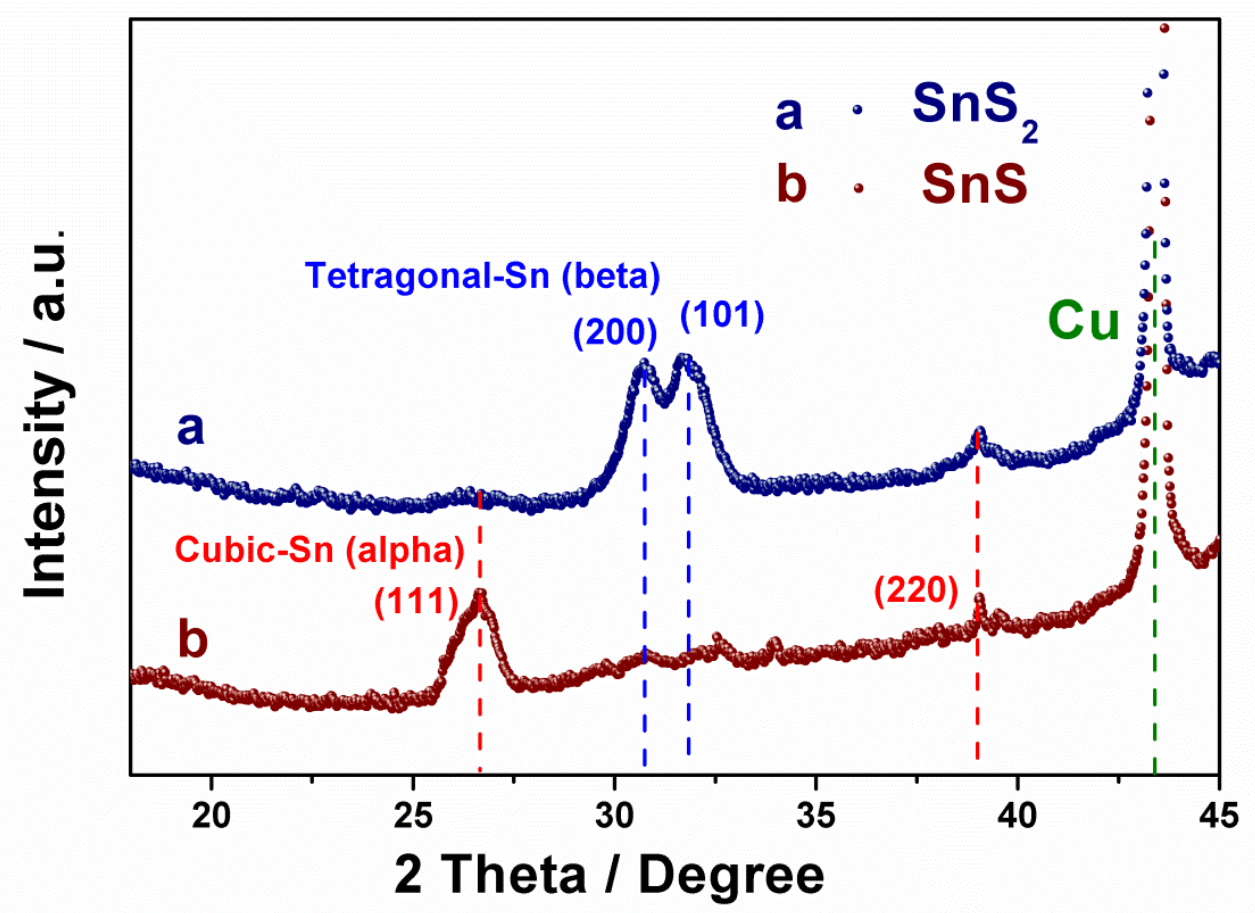

Figure 6. XRD patterns of the (a) $\mathrm{SnS}_{2}$ and (b) $\mathrm{SnS}$ electrodes collected at after the $50^{\text {th }}$ charge to $2.0 \mathrm{~V}$.

Meanwhile, stable cycling of an electrode has strict requirements in terms of structural stability at the particle level, because even small changes in particle morphology could accumulate across the thickness of the electrode and cause electrode-level cracking and failure. After 20 deep cycles at the current density of $270 \mathrm{~mA} \mathrm{~g}^{-1}$, the morphology of the SnS@graphene was examined with SEM. As the SEM micrographs in Figure S7 demonstrate, intact graphene sheets with SnS inside are clearly visible. There are two interdependent characteristics of the two-layered compound design that enable superior battery performance. The first is the internally accommodated volume expansion and the structural compatibility within the two-layered hybrid SnS@graphene architecture, which retains the structural 
integrity of the secondary particles. The second characteristic is the porous SnS@graphene structure, which can effectively absorb the stress that is generated during charge and discharge. The reduced charge-transfer resistance compared to that of the $\mathrm{SnS}_{2} @$ graphene and bare $\mathrm{SnS}_{2}$ electrodes is another factor, proving the high reactivity of the SnS@graphene electrode [Figure 7(a)]. This mechanism not only significantly stabilizes the mechanical, electrical, and electrochemical properties of the anode material, but also enables the inner void space to be retained.

Investigation of isolated active materials is no longer sufficient to solve all the different kinds of challenges for the development of large-scale energy storage. ${ }^{53}$ In this work, we have optimized the composite electrode by using high tensile-strength binders, polyacrylic acid (PAA) and sodium carboxymethyl cellulose (CMC), both of which were used to improve the cycling performance of the Liion battery electrode materials with large volume expansion. ${ }^{54,55}$ Compared to the electrode using PAACMC as binder, the electrode prepared with polyvinylidene difluoride (PVdF) exhibited severe capacity fading, which gave it much poorer performance than the cycling performance obtained with PAA-CMC binder [Figure 7(b)]. Additives such as fluoroethylene carbonate (FEC), which is known for stabilizing the SEI film in Na ion batteries, ${ }^{56}$ were also introduced into the electrolyte [Figure 7(c)] in order to further improve its electrochemical performance. 


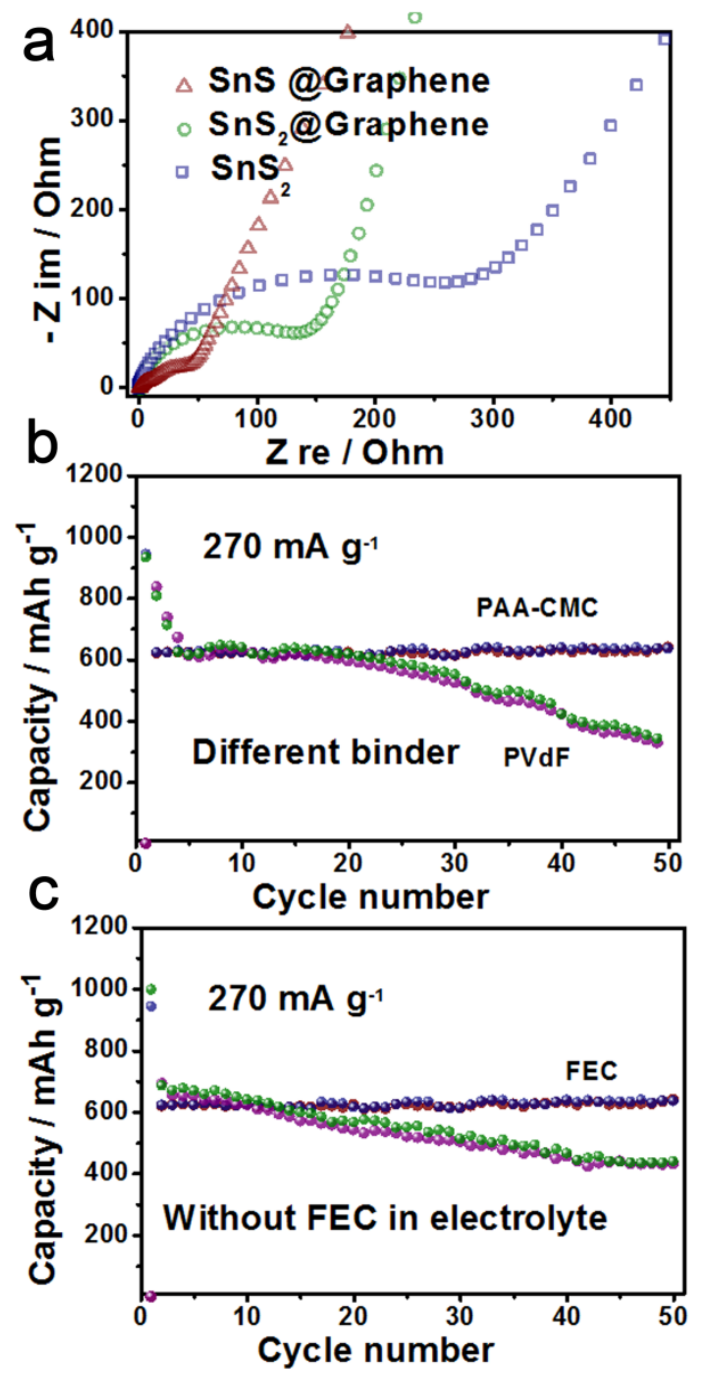

Figure 7. (a) Nyquist plots of electrodes containing hybrid SnS@graphene and $\mathrm{SnS}_{2} @ g$ graphene architectures, as well as bare $\mathrm{SnS}_{2}$, obtained by applying a sine wave with an amplitude of $5.0 \mathrm{mV}$ over the frequency range of $100 \mathrm{kHz}-0.01 \mathrm{~Hz}$. Representative cycling performances of electrodes with hybrid SnS@graphene architecture (b) with different binders and (c) with or without FEC in the electrolyte.

After systematic design and optimization of the active materials, buffer layer, binder, conductive additive, and electrolyte, the capacity and high-rate performance of the SnS@graphene architecture are much better than for most reported anode materials for Na ion batteries. Such extraordinary performance and excellent capacity retention at high charge-discharge rates suggest that the hybrid SnS@graphene 
architecture has great potential for the anode material in Na-ion batteries. The unprecedented excellent electrochemical performance of SnS@graphene can be attributed to the following reasons: (1) the structural compatibility within the two-layered hybrid SnS@graphene architecture and the excellent conductive electronic properties of the graphene network lead to very stable composites; (2) the porous structure of the composite electrode provides numerous channels to give access to the electrolyte, and the active framework made from filmy 2D SnS nanosheets provides a shortened ion diffusion path length; (3) the porous SnS@graphene structure can effectively absorb the stress which is generated during charge and discharge, while the structural stability of SnS layer structure should also be viable for reversible $\mathrm{Na}^{+}$storage, since in comparison to tin and other tin-based materials, it provides more buffering for the volume changes in Na-Sn reactions, so that acting together, they can perfectly maintain the structural stability of the whole electrode.

\section{Conclusions}

In summary, a hybrid SnS@graphene architecture, employing 2D nanosheets of SnS and graphene as complementary building blocks via their controllable assembly, was fabricated by a hydrothermal and annealing approach. The well-developed 2D nanosheets of SnS and graphene, as well as the precise hierarchical control of various sublayers of the materials in the design are believed to function synergistically, so as to significantly stabilize the mechanical, electrical, and electrochemical properties of the anode material, despite its large volume changes with sodiation/desodiation during cycling. From the perspective of structural ability during cycling, the less structural changes of SnS after the conversion, expected a good structural stability and excellent cycling stability for the performance on the sodium ion battery. The resultant architecture can provide high reversible capacity and excellent high-rate capability. The superior cycling and rate performance, combined with the simplicity of the 
optimized process, represents a promising strategy for the development of inexpensive and versatile synthesis techniques for energy storage and conversion applications.

\section{Experimental Section}

\section{Sample preparation}

Natural graphite powder was oxidized to graphite oxide by the modified Hummers' method. The SnS@graphene hybrid architecture was prepared by a hydrothermal route. In a typical batch, hexadecyltrimethyl ammonium bromide (CTAB, $0.218 \mathrm{~g}, 0.6 \mathrm{mmol}$ ) was dissolved in deionized (DI) water, and the as-prepared graphene oxide (GO, $4.0 \mathrm{mg} \mathrm{mL}^{-1}, 10 \mathrm{~mL}, 40 \mathrm{mg}$ ) was added into the solution. The mixture was stirred for another $2 \mathrm{~h}$ to allow $\mathrm{CTA}^{+}$adsorption on the GO surface by electrostatic interaction. After that, L-cysteine $(0.500 \mathrm{~g}, 4.0 \mathrm{mmol})$ and $\mathrm{K}_{2} \mathrm{SnO}_{3} \cdot 3 \mathrm{H}_{2} \mathrm{O}(0.300 \mathrm{~g}, 1.0$ mmol) were dissolved into the mixture, and DI water was added to adjust the volume of the mixture to $40 \mathrm{ml}$. After ultrasonication and stirring for $20 \mathrm{~min}$, the mixed suspension was transferred into a $50 \mathrm{~mL}$ Teflon-lined stainless steel autoclave, sealed tightly, and heated to $200{ }^{\circ} \mathrm{C}$ for $24 \mathrm{~h}$. After cooling naturally, the black solid product was collected by centrifugation, washed several times with DI water and ethanol, and dried in a vacuum oven at $60^{\circ} \mathrm{C}$ for $24 \mathrm{~h}$. The as-prepared product was then annealed in a tube furnace at $600{ }^{\circ} \mathrm{C}$ for $2 \mathrm{~h}$ in flowing argon. For the control experiment, $\mathrm{SnS}_{2}$ was also prepared by the above procedure without the presence of GO nanosheets.

\section{Characterization}

The crystalline structure of the as-prepared materials and ex-situ XRD were characterized by X-ray diffraction (XRD, MMA GBC). The morphologies and particle sizes of the samples were observed by field emission scanning electron microscopy (FESEM; JEOL-7500). The details of the crystal structure 
were further characterized by transmission electron microscopy (TEM), which was conducted on a JEOL-2010 transmission electron microscope operating at $200 \mathrm{kV}$. Selected area electron diffraction (SAED) patterns were recorded using a Gatan charge coupled device (CCD) camera in a digital format. X-ray photoelectron spectroscopy (XPS) was conducted on a VG Multilab 2000. All of the binding energies were referenced to the $\mathrm{C} 1 \mathrm{~s}$ peak at $284.8 \mathrm{eV}$ of the surface adventitious carbon. Thermogravimetric analysis (TGA) was conducted on a TA 2000 Thermo-analyzer.

\section{Electrochemical measurements}

The electrochemical tests were carried out via CR2032 coin type cells. The working electrodes were prepared by mixing the as-prepared materials, Super P, sodium carboxymethyl cellulose / polyacrylic acid (1:1) at a weight ratio of 80:10:10. The resultant slurry was pasted on $\mathrm{Cu}$ foil and dried in a vacuum oven at $150{ }^{\circ} \mathrm{C}$ for $3 \mathrm{~h}$, followed by pressing at $300 \mathrm{~kg} \mathrm{~cm}^{-2}$. The weight of the materials on individual electrodes was $1.0 \pm 0.2 \mathrm{mg} \mathrm{cm}^{-2}$. Electrochemical measurements were carried out using two-electrode coin cells with Na metal as counter and reference electrode and glass microfiber (Whatman) as the separator. The electrolyte consisted of a solution of $1 \mathrm{M} \mathrm{NaClO}_{4}$ in ethylene carbonate (EC) / propylene carbonate (PC) $(1 / 1 ; \mathrm{v} / \mathrm{v})$ with 5 wt\% fluoroethylene carbonate (FEC). Electrochemical impedance spectroscopy (EIS) and cyclic voltammetry (CV) were conducted on a VMP-3 electrochemical workstation at a scan rate of $0.1 \mathrm{mV} \mathrm{s}^{-1}$. The cells were galvanostatically charged and discharged over a voltage range of $0.01-3 \mathrm{~V}$ versus $\mathrm{Na} / \mathrm{Na}^{+}$at different constant current densities, based on the weight of the samples, on a Land CT2001A battery tester. At least five parallel cells were tested for each electrochemical measurement, in order to make sure that the results were reliable and represented the typical behavior of the samples.

\section{Supporting Information Available:}


Detailed Fourier transform infrared (FTIR) spectra, XPS spectra, TGA curve, EDS spectra, and coulombic efficiency characterizations. This material is available free of charge via the internet at http:// pubs.acs.org.

\section{Corresponding Author}

Zaiping Guo ${ }^{\mathrm{a}, \mathrm{b}}$

a Institute for Superconducting and Electronic Materials, School of Mechanical, Materials and Mechatronics Engineering, University of Wollongong, North Wollongong, NSW 2500, Australia.

b Hubei Collaborative Innovation Center for Advanced Organic Chemical Materials, College of Chemistry and Chemical Engineering, Hubei University, Wuhan 430062, P.R. China.

E-mail: zguo@uow.edu.au

\section{AUTHOR CONTRIBUTIONS}

The manuscript was written through the contributions of all the authors. All authors have given approval to the final version of the manuscript.

\section{FUNDING SOURCES}

The Australian Research Council (ARC) through an ARC Discovery project (DP1094261) is gratefully acknowledged.

\section{ACKNOWLEDGMENTS}

Financial support provided by the Australian Research Council (ARC) through an ARC Discovery project (DP1094261) is gratefully acknowledged. The authors would also like to thank the Electron Microscopy Centre (EMC) at the University of Wollongong for the electron microscopy characterizations. The authors would like to thank Dr. Kuok Hau Seng, and Dr. Li Li for their help, and also thank Dr. T. Silver for critical reading of the manuscript. 


\section{REFERENCES}

1. Bruce, P. G.; Scrosati, B.; Tarascon, J. M. Nanomaterials for Rechargeable Lithium Batteries. Angew. Chem. Int. Edit. 2008, 47, 2930-2946.

2. Yang, Z. G.; Zhang, J. L.; Kintner-Meyer, M. C. W.; Lu, X. C.; Choi, D. W.; Lemmon, J. P.; Liu, J. Electrochemical Energy Storage for Green Grid. Chem. Rev. 2011, 111, 3577-3613.

3. Liu, J.; Zhang, J. G.; Yang, Z. G.; Lemmon, J. P.; Imhoff, C.; Graff, G. L.; Li, L. Y.; Hu, J. Z.; Wang, C. M.; Xiao, J.; Xia, G.; Viswanathan, V. V.; Baskaran, S.; Sprenkle, V.; Li, X. L.; Shao, Y. Y.; Schwenzer, B. Materials Science and Materials Chemistry for Large Scale Electrochemical Energy Storage: From Transportation to Electrical Grid. Adv. Funct. Mater. 2013, 23, 929-946.

4. Kim, S. W.; Seo, D. H.; Ma, X. H.; Ceder, G.; Kang, K. Electrode Materials for Rechargeable Sodium-Ion Batteries: Potential Alternatives to Current Lithium-Ion Batteries. Adv. Energy Mater. 2012, 2, 710-721.

5. $\quad$ Ellis, B. L.; Makahnouk, W. R. M.; Makimura, Y.; Toghill, K.; Nazar, L. F. A Multifunctional 3.5 V Iron-based Phosphate Cathode for Rechargeable Batteries. Nat. Mater. 2007, 6, 749-753.

6. Lu, Y. H.; Wang, L.; Cheng, J. G.; Goodenough, J. B. Prussian Blue: A New Framework of Electrode Materials for Sodium Batteries. Chem. Commun. 2012, 48, 6544-6546.

7. Ong, S. P.; Chevrier, V. L.; Hautier, G.; Jain, A.; Moore, C.; Kim, S.; Ma, X. H.; Ceder, G. Voltage, Stability and Diffusion Barrier Differences Between Sodium-ion and Lithium-ion Intercalation materials. Energy Environ. Sci. 2011, 4, 3680-3688.

8. Wang, J. W.; Liu, X. H.; Mao, S. X.; Huang, J. Y. Microstructural Evolution of Tin Nanoparticles during In Situ Sodium Insertion and Extraction. Nano Lett. 2012, 12, 5897-5902. 
9. Xu, Y. H.; Zhu, Y. J.; Liu, Y. H.; Wang, C. S. Electrochemical Performance of Porous Carbon/Tin Composite Anodes for Sodium-Ion and Lithium-Ion Batteries. Adv. Energy Mater. 2013, 3, 128-133.

10. Liu, Y. H.; Xu, Y. H.; Zhu, Y. J.; Culver, J. N.; Lundgren, C. A.; Xu, K.; Wang, C. S. TinCoated Viral Nanoforests as Sodium-Ion Battery Anodes. ACS Nano 2013, 7, 3627-3634.

11. Xiao, L. F.; Cao, Y. L.; Xiao, J.; Wang, W.; Kovarik, L.; Nie, Z. M.; Liu, J. High Capacity, Reversible Alloying Reactions in SnSb/C Nanocomposites for Na-ion Battery Applications. Chem. Commun. 2012, 48, 3321-3323.

12. Park, J.; Kim, J.-S.; Park, J.-W.; Nam, T. H.; Kim, K. W.; Ahn, J. H.; Wang, G.; Ahn, H. J. Discharge Mechanism of $\mathrm{MoS}_{2}$ for Sodium Ion Battery: Electrochemical Measurements and Characterization. Electrochimica Acta 2013, 92, 427-432.

13. Lu, J.; Nan, C. Y.; Li, L. H.; Peng, Q.; Li, Y. D. Flexible SnS Nanobelts: Facile Synthesis, Formation Mechanism and Application in Li-ion Batteries. Nano Res. 2013, 6, 55-64.

14. Seo, J. W.; Jang, J. T.; Park, S. W.; Kim, C. J.; Park, B. W.; Cheon, J. W. Two-Dimensional $\mathrm{SnS}_{2}$ Nanoplates with Extraordinary High Discharge Capacity for Lithium Ion Batteries. Adv. Mater. 2008, 20, 4269-4273.

15. Mahmood, N.; Zhang, C. Z.; Hou, Y. L. Nickel Sulfide/Nitrogen-Doped Graphene Composites: Phase-Controlled Synthesis and High Performance Anode Materials for Lithium Ion Batteries. Small 2013, 9, 1321-1328.

16. Huang, G.; Chen, T.; Chen, W.; Wang, Z.; Chang, K.; Ma, L.; Huang, F.; Chen, D.; Lee, J. Y. Graphene-Like $\mathrm{MoS}_{2} /$ Graphene Composites: Cationic Surfactant-Assisted Hydrothermal Synthesis and Electrochemical Reversible Storage of Lithium. Small 2013, 9, 3693-3703. 
17. Zhang, Y. W.; Tian, J. Q.; Li, H. Y.; Wang, L.; Qin, X. Y.; Asiri, A. M.; Al-Youbi, A. O.; Sun, X. P. Biomolecule-Assisted, Environmentally Friendly, One-Pot Synthesis of CuS/Reduced Graphene Oxide Nanocomposites with Enhanced Photocatalytic Performance. Langmuir 2012, 28, 12893-12900.

18. Qu, B.; Ma, C.; Ji, G.; Xu, C.; Xu, J.; Meng, Y. S.; Wang, T.; Lee, J. Y. Layered SnS 2 -Reduced Graphene Oxide Composite - A High-Capacity, High-Rate, and Long-Cycle Life Sodium-Ion Battery Anode Material. Adv. Mater. 2014, 26, 3854-3859.

19. Xie, X.; Su, D.; Chen, S.; Zhang, J.; Dou, S.; Wang, G. SnS 2 Nanoplatelet@Graphene Nanocomposites as High-Capacity Anode Materials for Sodium-Ion Batteries. Chemistry - An Asian Journal 2014, 9, 1611-1617.

20. David, L.; Bhandavat, R.; Singh, G. $\mathrm{MoS}_{2}$ /Graphene Composite Paper for Sodium-Ion Battery Electrodes. ACS Nano 2014, 8, 1759-1770.

21. Sathish, M.; Mitani, S.; Tomai, T.; Honma, I. Ultrathin $\mathrm{SnS}_{2}$ Nanoparticles on Graphene Nanosheets: Synthesis, Characterization, and Li-Ion Storage Applications. J. Phys. Chem. C 2012, 116, $12475-12481$.

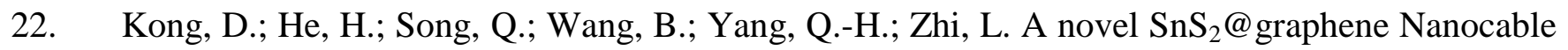
Network for High-performance Lithium Storage. RSC Advances 2014, 4, 23372-23376.

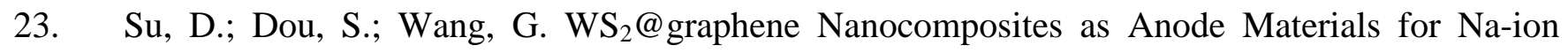
Batteries with Enhanced Electrochemical Performances. Chem. Commun. 2014, 50, 4192-4195.

24. Deng, Z. T.; Cao, D.; He, J.; Lin, S.; Lindsay, S. M.; Liu, Y. Solution Synthesis of Ultrathin Single-Crystalline SnS Nanoribbons for Photodetectors via Phase Transition and Surface Processing. ACS Nano 2012, 6, 6197-6207.

25. Zhang, Y. J.; Lu, J.; Shen, S. L.; Xu, H. R.; Wang, Q. B. Ultralarge Single Crystal SnS Rectangular Nanosheets. Chem. Commun. 2011, 47, 5226-5228. 
26. Cao, T.; Wang, G.; Han, W.; Ye, H.; Zhu, C.; Shi, J.; Niu, Q.; Tan, P.; Wang, E.; Liu, B.; Feng, J. Valley-selective Circular Dichroism of Monolayer Molybdenum Disulphide. Nat Commun 2012, 3, 887.

27. Zeng, H.; Dai, J.; Yao, W.; Xiao, D.; Cui, X. Valley Polarization in $\mathrm{MoS}_{2}$ Monolayers by Optical Pumping. Nat Nano 2012, 7, 490-493.

28. Mak, K. F.; He, K.; Shan, J.; Heinz, T. F. Control of Valley Polarization in Monolayer MoS 2 by Optical helicity. Nat Nano 2012, 7, 494-498.

29. Wei, R.; Hu, J.; Zhou, T.; Zhou, X.; Liu, J.; Li, J. Ultrathin $\mathrm{SnS}_{2}$ Nanosheets with Exposed $\{001\}$ Facets and Enhanced Photocatalytic Properties. Acta Materialia 2014, 66, 163-171.

30. Wei, R.; Zhou, T.; Hu, J.; Li, J. Glutatione Modified Ultrathin $\mathrm{SnS}_{2}$ Nanosheets with Highly Photocatalytic Activity for Wastewater Treatment. Mater. Res. Exp. 2014, 1, 025018.

31. Biacchi, A. J.; Vaughn, D. D.; Schaak, R. E. Synthesis and Crystallographic Analysis of ShapeControlled SnS Nanocrystal Photocatalysts: Evidence for a Pseudotetragonal Structural Modification. J. Am. Chem. Soc. 2013, 135, 11634-11644.

32. Meyer, J. C.; Geim, A. K.; Katsnelson, M. I.; Novoselov, K. S.; Booth, T. J.; Roth, S. The structure of suspended graphene sheets. Nature 2007, 446, 60-63.

33. Bertolazzi, S.; Brivio, J.; Kis, A. Stretching and Breaking of Ultrathin $\mathrm{MoS}_{2}$. ACS Nano 2011, 5, 9703-9709.

34. Zhang, B.; Ye, X. C.; Dai, W.; Hou, W. Y.; Xie, Y. Biomolecule-assisted Synthesis and Electrochemical Hydrogen Storage of Porous Spongelike $\mathrm{Ni}_{3} \mathrm{~S}_{2}$ Nanostructures Grown Directly on Nickel Foils. Chem.-Eur. J. 2006, 12, 2337-2342.

35. Chang, K.; Chen, W. X. L-Cysteine-Assisted Synthesis of Layered $\mathrm{MoS}_{2} /$ Graphene Composites with Excellent Electrochemical Performances for Lithium Ion Batteries. ACS Nano 2011, 5, 4720-4728. 
36. Chhowalla, M.; Shin, H. S.; Eda, G.; Li, L.-J.; Loh, K. P.; Zhang, H. The Chemistry of TwoDimensional Layered Transition Metal Dichalcogenide Nanosheets. Nat Chem 2013, 5, 263-275.

37. Gong, Y.; Yang, S.; Liu, Z.; Ma, L.; Vajtai, R.; Ajayan, P. M. Graphene-network-backboned Architectures for High-performance Lithium Storage. Adv. Mater. 2013, 25, 3979-84.

38. Fasolino, A.; Los, J. H.; Katsnelson, M. I. Intrinsic Ripples in Graphene. Nat. Mater. 2007, 6, 858-861.

39. Chen, Z.; Yuan, Y.; Zhou, H.; Wang, X.; Gan, Z.; Wang, F.; Lu, Y. 3D Nanocomposite Architectures from Carbon-Nanotube-Threaded Nanocrystals for High-Performance Electrochemical Energy Storage. Adv. Mater. 2014, 26, 339-345.

40. Arico, A. S.; Bruce, P.; Scrosati, B.; Tarascon, J. M.; Van Schalkwijk, W. Nanostructured Materials for Advanced Energy Conversion and Storage Devices. Nat. Mater. 2005, 4, 366-377.

41. Xiang, J. H.; Cao, H. Q.; Wu, Q. Z.; Zhang, S. C.; Zhang, X. R.; Watt, A. A. R. L-CysteineAssisted Synthesis and Optical Properties of $\mathrm{Ag}_{2} \mathrm{~S}$ Nanospheres. J. Phys. Chem. C 2008, 112, 35803584.

42. Liu, Y.; Ma, W. S.; Liu, W. W.; Li, C.; Liu, Y. L.; Jiang, X. Y.; Tang, Z. Y. Silver(I)-glutathione Biocoordination Polymer Hydrogel: Effective Antibacterial Activity and Improved Cytocompatibility. J. Mater. Chem. 2011, 21, 19214-19218.

43. Xu, Y.; Zhao, W. W.; Xu, R.; Shi, Y. M.; Zhang, B. Synthesis of Ultrathin CdS Nanosheets as Efficient Visible-light-driven Water Splitting Photocatalysts for Hydrogen Evolution. Chem. Commun. 2013, 49, 9803-9805.

44. Aso, K.; Kitaura, H.; Hayashi, A.; Tatsumisago, M. Synthesis of Nanosized Nickel Sulfide in High-boiling Solvent for All-solid-state Lithium Secondary Batteries. J. Mater. Chem. 2011, 21, 29872990. 
45. Fernandes, P. A.; Sousa, M. G.; Salome, P. M. P.; Leitao, J. P.; da Cunha, A. F. Thermodynamic Pathway for the Formation of SnSe and $\mathrm{SnSe}_{2}$ Polycrystalline Thin Films by Selenization of Metal Precursors. Crystengcomm 2013, 15, 10278-10286.

46. Wiedemeier, H.; Pultz, G. Equilibrium Sublimation and Thermodynamic Properties of SnSe and SnSe 2 . Zeit. Anorgan. Allgeme. Chem. 1983, 499, 130-144.

47. Zhou, X. L.; Zhou, T. F.; Hu, J. C.; Li, J. L. Controlled Strategy to Synthesize $\mathrm{SnO}_{2}$ Decorated $\mathrm{SnS}_{2}$ Nanosheets with Enhanced Visible Light Photocatalytic Activity. CrystEngComm 2012, 14, 56275633.

48. Luo, B.; Fang, Y.; Wang, B.; Zhou, J. S.; Song, H. H.; Zhi, L. J. Two Dimensional Graphene$\mathrm{SnS}_{2}$ Hybrids with Superior Rate Capability for Lithium Ion Storage. Energy Environ. Sci. 2012, 5, 5226-5230.

49. Chevrier, V. L.; Ceder, G. Challenges for Na-ion Negative Electrodes. J. Electrochem. Soc. 2011, 158, A1011-A1014.

50. Im, H. S.; Cho, Y. J.; Lim, Y. R.; Jung, C. S.; Jang, D. M.; Park, J.; Shojaei, F.; Kang, H. S. Phase Evolution of Tin Nanocrystals in Lithium Ion Batteries. ACS Nano 2013.

51. $\quad$ Cho, Y. J.; Im, H. S.; Kim, H. S.; Myung, Y.; Back, S. H.; Lim, Y. R.; Jung, C. S.; Jang, D. M.; Park, J.; Cha, E. H.; Cho, W. I.; Shojaei, F.; Kang, H. S. Tetragonal Phase Germanium Nanocrystals in Lithium Ion Batteries. ACS Nano 2013, 7, 9075-9084.

52. Yu, D. Y. W.; Prikhodchenko, P. V.; Mason, C. W.; Batabyal, S. K.; Gun, J.; Sladkevich, S.; Medvedev, A. G.; Lev, O. High-capacity Antimony Sulphide Nanoparticle-decorated Graphene Composite as Anode for Sodium-ion Batteries. Nat Commun 2013, 4. 
53. Wang, L.; Wang, D.; Dong, Z.; Zhang, F.; Jin, J. Interface Chemistry Engineering of ProteinDirected $\mathrm{SnO}_{2}$ Nanocrystal-Based Anode for Lithium-Ion Batteries with Improved Performance. Small 2014, 10, 998-1007.

54. Sun, Y.; Zhao, L.; Pan, H. L.; Lu, X.; Gu, L.; Hu, Y. S.; Li, H.; Armand, M.; Ikuhara, Y.; Chen, L. Q.; Huang, X. J. Direct Atomic-scale Confirmation of Three-phase Storage Mechanism in $\mathrm{Li}_{4} \mathrm{Ti}_{5} \mathrm{O}_{12}$ Anodes for Room-temperature Sodium-ion Batteries. Nat. Commun. 2013, 4.

55. Koo, B.; Kim, H.; Cho, Y.; Lee, K. T.; Choi, N. S.; Cho, J. A Highly Cross-Linked Polymeric Binder for High-Performance Silicon Negative Electrodes in Lithium Ion Batteries. Angew. Chem. Int. Edit. 2012, 51, 8762-8767.

56. Oh, S. M.; Myung, S. T.; Jang, M. W.; Scrosati, B.; Hassoun, J.; Sun, Y. K. An Advanced Sodium-ion Rechargeable Battery Based on a Tin-carbon Anode and a Layered Oxide Framework Cathode. Phys. Chem. Chem. Phys. 2013, 15, 3827-3833. 
Cover Graphics

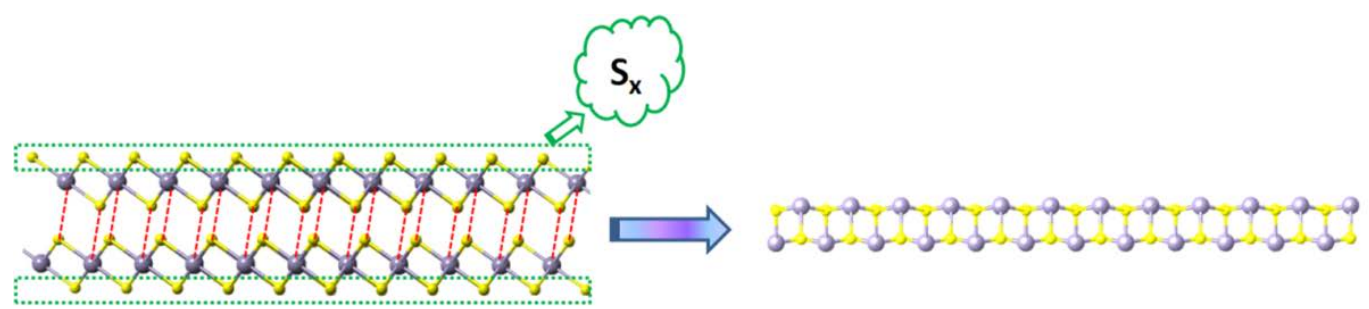

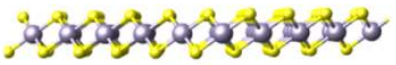

roros $\operatorname{SnS}_{2}(\mathrm{~s}) \stackrel{\Delta}{\rightarrow} \mathrm{SnS}(\mathrm{s})+\frac{1}{\mathrm{x}} \mathrm{S}_{\mathrm{x}}(\mathrm{g})$ aces

Phase Transition

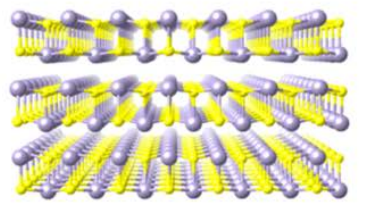

SnS 2 Hexagonal

Orthorhombic SnS

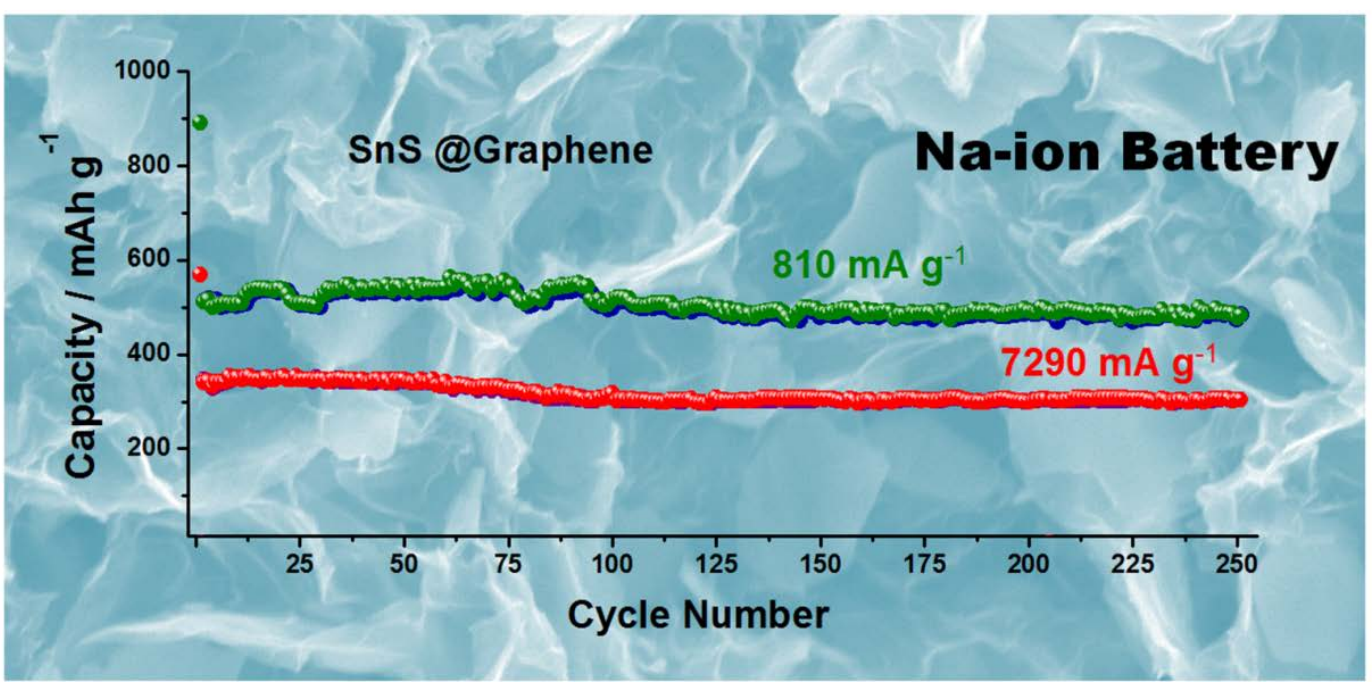

